\title{
25 Research Square \\ TLK1-MK5 axis drives prostate cancer cell motility and pathologic features of aggressiveness
}

\section{Md Imtiaz Khalil}

LSUHSC-S: LSU Health Shreveport

\section{Vibha Singh}

LSUHSC-S: LSU Health Shreveport

Judy King

LSUHSC Shreveport: LSU Health Shreveport

Arrigo De Benedetti ( $\nabla$ adeben@lsuhsc.edu )

"Louisiana State University Health Sciences Center Shreveport" https://orcid.org/0000-0002-4198-8647

\section{Research Article}

Keywords: TLK1, MK5, cell motility, prostate cancer metastasis, PCa TMA

Posted Date: April 27th, 2021

DOl: https://doi.org/10.21203/rs.3.rs-434116/v1

License: (9) This work is licensed under a Creative Commons Attribution 4.0 International License. Read Full License 


\section{Abstract}

Background: Majority of prostate cancer ( $\mathrm{PCa}$ ) related fatalities occur due to metastasis of cancer cells to adjacent and distal organs. We identified the novel interaction between two kinases (TLK1-MK5) that in part may initiate a signaling cascade promoting PCa metastasis. In PCa, TLK1-MK5 signaling might be crucial as androgen deprivation therapy leads to increased expression of TLK1 and compensatory activation of MK5 in metastatic castration-resistant prostate cancer patients.

Methods: We performed scratch wound repair and 3D chemotactic migration assays to determine the motility rates of different TLK1 and MK5 perturbed cells. Co-IP, His, and GST pull down, in vitro kinase (IVK) assays and mass spectrometry (MS) were conducted to determine TLK1-MK5 interaction and phosphorylation. Western blotting (WB), immunohistochemistry (IHC) and bioinformatic analysis were used to examine TLK1 and pMK5 levels in PCa cell lines, mice prostate tumors and PCa tissue microarray (TMA).

Results: Both genetic depletion and pharmacologic inhibition of TLK1 and MK5 can significantly reduce wound healing rate in MEF and LNCaP cells. However, TLK1 overexpression alone in the MK5 -/- MEF cells did not increase the wound healing which suggested that TLK1 cannot enhance cellular migration in absence of MK5. Our reciprocal co-IPs, His- and GST pull down assays confirmed TLK1-MK5 interaction in cultured cells. Incubation of purified recombinant TLK1B and MK5 increases the phosphorylation of MK5 and its kinase activity. MS analysis identified three unique phosphorylation sites in MK5 (S160, S354, S386) by TLK1B. While our WB detected substantial amount of pMK5 S354 and TLK1 in all major PCa cell lines, anti-androgen treatment increased pMK5 S354 level in a dose-dependent manner and pharmacologic inhibition of TLK1 reduces pMK5 S354 level in LNCaP cells. IHC staining of TRAMP mice prostate tissues also exhibited increased pMK5 S354 level in aggressive tumor compared to benign regions. Finally, IHC analysis of PCa TMA indicated a correlation between elevated pMK5 S354 level and generally higher Gleason scores as well as nodal metastatic status of the tumors.

Conclusion: Our data support that TLK1-MK5 signaling is functionally involved in driving PCa cell motility and clinical aggressiveness, hence, disruption of this axis may inhibit the metastasis of PCa.

\section{Introduction}

Prostate cancer (PCa) is the 2nd leading death causing malignancy in men in the United States [1]. Like other malignancies, metastasis has been the primary cause of PCa related deaths. PCa patients with distant organ metastasis have a 5-years survival rate of only around $30 \%$ [2] and currently, no curative therapy is available for the treatment of metastatic PCa [3]. Androgen deprivation therapy (ADT) has been the gold standard method to treat PCa. However, after 1-2 years of treatment, the tumor relapses with more aggressive phenotype, known as metastatic castration resistant PCa (mCRPC). Cellular motility and invasion are the prerequisites for the cancer cells to migrate and localize in the adjacent as well as distant organs. Cell migration and invasion are multi-factor processes involving numerous signaling 
pathways. Thus, identification of these factors and underlying signaling cascades is required to devise a therapeutic intervention which may reduce the spread of the cancer cells.

Tousled kinase (TSL) was first discovered in Arabidopsis thaliana and found crucial for leaf and flower development of the plant [4,5]. Later, homologues of TSL were identified in most metazoans which are named as tousled-like kinase (TLK) [6]. TLKs are serine/threonine kinases, with maximal activity in the Sphase of the cell cycle, play roles in replication, transcription, chromosomal segregation, DNA damage response and repair. In mammals, two genes are present in the tousled like kinase family- Tlk1 and Tlk2 which share $89 \%$ homology in their entire amino acid sequence and $94 \%$ similarity in their C-terminal kinase domain (rev. in [7]). Each gene encodes several isoforms of which TLK1B, a spliced variant with long untranslated region and two regulatory uORFs, is well characterized by our group [8]. Histone H3, histone chaperone ASF1, RAD9, and NEK1 are some of the well-validated substrates of TLK1, which can be directly phosphorylated by TLK1/1B. The activating phosphorylation of NEK1 on T141 residue by TLK1 results in the initiation of DNA damage response signaling through TLK1 > NEK1 > ATR > CHK1 axis that contributes to castration resistant progression of $\mathrm{PCa}[9,10]$. In addition, recent findings suggest that TLK1 mediated activation of NEK1 also leads to apoptotic prevention and YAP stabilization which facilitates cancer cell survival and conversion to androgen independence [11, 12]. Recently, two research groups independently reported that TLK2 enhances the migration rate and invasiveness of breast cancer and glioblastoma cell lines and hence, can be considered a factor for aggressiveness [13, 14]. Another study revealed that the gene encoding tousled like kinase (TIk) in Drosophila (Drosophila has only one TIk gene) is required for the collective migration of border cells by activating JAK/STAT signaling pathway in the ovary, a phenomenon that is reminiscent of cancer migration/metastasis [15]. Since TLK1 and TLK2 have higher sequence similarity and function similarly, we hypothesized that TLK1 may also play role in the dissemination of the cancer cells.

The 2 nd protein player in this study is MAPK-activated protein kinase 5 (MAPKAPK5 or MK5), a serine/threonine kinase that has been studied in several neoplastic and non-malignant cells. MK5 can be activated by both atypical and typical upstream MAP kinase, ERK3/4 and p38, respectively. Hence, it has acquired the acronym PRAK (p38 regulated and activated kinase). MK5 is known to be a genuine promotility factor that drives cell motility by regulating actin cytoskeletal rearrangements and focal adhesion complex modification [16-21]. However, a few other studies suggested the antimotility role of MK5 in HeLa and U2OS osteosarcoma cells where MK5 exerts its function through the impairment of FAK activation and filamentous actin formation respectively $[22,23]$. MK5's role in motility is thus dependent on the cellular context, type and stage of the cancer. Other biological functions of MK5 includes cell growth, oncogene induced senescence, transcriptional regulation, neuronal spine formation, cellular survival, and regulation of cardiac fibroblast function [19, 24-33]. Out of 11 members in the MAPKAPK family, MK5 is most closely related to MK2 and MK3 and shares approximately $33 \%$ sequence similarity (rev. in [34-37]). Although MK2 and MK5 have several common substrates, their functions are temporally and spatially separated [17]. MK5 can act as both tumor suppressor and promoter [16, 25, 36, 38]. For instance, MK5 is reported to negatively regulate Myc transcription and positively regulate YAP's stability by inhibiting its degradation $[24,39]$. MK5's implication in PCa metastasis has not been characterized yet. 
Here we describe the potential implications of TLK1-MK5 signaling in prostate cancer cell motility and whether disruption of this axis can inhibit PCa cell migration. Our data suggest that MK5 is a direct substrate of TLK1 which can be phosphorylated on several serine residues. We also establish MK5 as a genuine promotility factor in PCa cell lines and suggest that TLK1 phosphorylation of MK5 may enhance its pro-motility activity. Our bioinformatic study also suggested a correlation of TLK1 and MK5 expression with the aggressiveness of prostate tumors which hints toward a clinical relevance of TLK1-MK5 signaling in PCa metastasis. In addition, IHC analysis of a PCa TMA revealed that high pMK5-S354 stain correlated with presence on local nodal involvement in biopsies.

\section{Materials And Methods}

\section{Plasmids and antibodies}

Wild type human full length TLK1 mammalian expression plasmid was purchased from Addgene (Watertown, MA, USA, cat\# 98378). Wild type full length human TLK1B bacterial expression plasmid was obtained from Dr. Sivapriya Kirubakaran, Discipline of Biological Engineering, Indian Institute of Technology, Gandhinagar, India [40]. Dominant negative human TLK1 kinase dead mammalian expression vector was generated as previously described [41]. pEGFP-MK5-C1 mammalian expression vector was a kind gift from Dr. Ole Morten Seternes (Department of Pharmacology, 1 Institute of Medical Biology, University of Troms $\varnothing$, Troms $\varnothing$, Norway) [42]. Human full length MK5 bacterial expression plasmid was purchased from Vector Builder (Chicago, IL, USA). The following primary antibodies were used in this study: rabbit anti-TLK1 (ThermoFisher, Waltham, MA, USA, cat\# 720397), rabbit anti-TLK1B (lab generated), rabbit anti-MK5 (Cell Signaling technology, CST, Danvers, MA, USA, cat\# 7419S), mouse antiPRAK (Santa Cruz Biotechnology, SCBT, Dallas, TX, USA, sc-46667), rabbit anti phospho-MK5 S354 (ThermoFisher, cat\# PA5-105676), mouse anti-GFP (ThermoFisher, cat\# MA5-15256), rabbit anti-GAPDH (CST, cat\# 2118S), rabbit anti-actin (Abcam, Cambridge, MA, USA, cat\# ab1801).

\section{Cell culture}

Human embryonic kidney 293 (HEK 293) and mouse embryonic fibroblast (MEF) cells were cultured in DMEM supplemented with 10\% FBS and 1\% penicillin/streptomycin. PCa cell lines- LNCaP, C4-2B, PC3, DU145, and 22Rv1 were cultured in RPMI1640 supplemented with 10\% FBS and 1\%

Penicillin/streptomycin. VCaP cells were cultured according to ATCC provided method. MK5 ${ }^{-/-}$MEF cells were a kind gift from Dr. Matthias Gaestel, University of Hannover, Germany [43]. All the other cell lines were purchased from American Type Culture Collection (ATCC, Manassas, VA, USA) and authenticated within the past three years. All the cells were maintained in humidified incubator at $37^{\circ} \mathrm{C}$ with $5 \% \mathrm{CO}_{2}$.

\section{Cell treatment}

LNCaP cells were treated with $5 \mu \mathrm{M}$ or $10 \mu \mathrm{M}$ of bicalutamide (Selleckchem, Houston, TX, USA, cat\# S1190) or $10 \mu \mathrm{M}$ of thioridazine (THD; Sigma Aldrich, St. Louis, MO, USA, cat\# T9025) or J54 [44] for 48 hours in a 6-well plate when the confluency reached 70-90\%. DMSO treated LNCaP cells were considered 
as vehicle control (VC). After the treatment, cells were harvested for western blotting (WB) analysis. GFPMK5 and TLK1 co-expressing cell lysates was treated with lambda protein phosphatase (LPP) (New England Biolabs, Inc., Lincoln, NE, USA, cat\# P0753S) following manufacturer protocol.

\section{Cell transfection}

Wild type MEF (MEF WT) and LNCaP cells were transfected with eGFP-MK5 by lipofectamine 3000 (Thermo Scientific, Waltham, MA, USA, cat\# L3000-015) following manufacturer protocol and GFP positive cells were sort gated by flow cytometry. MK5 $5^{-/-}$MEF cells were transfected with eGFP-MK5 or TLK1 mammalian expression plasmid by lipofectamine 3000 and sort gated by flow cytometry or selected by $1-2 \mu \mathrm{g} / \mathrm{ml}$ puromycin treatment for seven days. LNCaP cells were also transfected with kinase dead TLK1 plasmid and selected by $400-500 \mu \mathrm{g} / \mathrm{ml} \mathrm{G418}$ for seven days. HEK293 cells were first transfected with eGFP-MK5 and selected by flow cytometry. MK5 positive cells were further transfected with either TLK1 or kinase dead TLK1 plasmid and selected by antibiotic treatment. To knockdown TLK1, MEF WT cells were plated as 40000 cells/well in a 6-well plate and grown until $60-70 \%$ confluency. Cells were transfected with two $100 \mathrm{nM}$ doses of mouse specific TLK1 siRNA within 24 hours apart (Thermo Scientific, Waltham, MA, USA, cat\# AM16708) by lipofectamine 3000. 72 hours after the 2nd dose of siRNA treatment, cells were split and harvested for scratch wound repair assay and WB analysis.

\section{Scratch wound repair assay}

Different MEF derivatives and PCa (C4-2B, PC3, DU145, and 22Rv1) cells were seeded as 40000 cells/well in a 96-well ImageLock plate (Essen Biosciences, Inc., Ann Arbor, MI, USA, cat\# 4379) in 10\% FBS containing media. LNCaP cells were seeded as $80000-90000$ cells/well in a 96-well ImageLock plate. After 48 hours when $90-100 \%$ confluency was achieved, cells were scratched by a wound maker (Essen Biosciences, Inc., cat\# 4493), washed twice with serum free media and supplemented with low serum (0.5-1\% FBS) media with or without treatment. Media was supplemented with either DMSO as vehicle control (VC) or $10 \mu \mathrm{M}$ J54 [44] or 5-20 $\mu \mathrm{M}$ of GLPG0259 (Medkoo Biosciences, Inc., Morrisville, NC, USA, cat\# 561481). The plates were then transferred to a Incucyte Zoom incubator with $5 \% \mathrm{CO}_{2}$ at $37^{\circ} \mathrm{C}$ and the migration of the cells were monitored by Incucyte zoom live cell imaging system (Essen Biosciences, Inc., Ann Arbor, MI, USA). Machine controlled images were taken every 4 hours to determine the relative wound density of each well. Mean rate (MR) was determined by calculating the slopes of the curve.

\section{D chemotactic trans-well migration assay}

Both sides of the porous membrane of the top insert of Incucyte clearview 96-well chemotaxis plate (Essen Biosciences, Inc., cat\# 4582) were coated with either fibronectin $(1 \mathrm{mg} / \mathrm{ml})$, or Matrigel $(50 \mu \mathrm{g} / \mathrm{ml})$, or Collagen I $(50 \mathrm{ug} / \mathrm{ml})$ three hours before seeding the cells. Cells were seeded in the top chamber as 1000 cells/well in low serum ( $0.5 \%$ FBS) media. Chemotactic gradient was created by putting $10 \%$ FBS containing media in the bottom reservoir. Cells were transferred to a Incucyte Zoom incubator with $5 \%$ $\mathrm{CO}_{2}$ at $37^{\circ} \mathrm{C}$ and the migration of the cells towards chemotactic gradient were monitored by Incucyte zoom live cell analysis system. Images wee taken at every four hours of both top insert and bottom reservoir and total cell occupancy in the bottom reservoir normalized to initial top value was determined. 


\section{Proliferation assay}

Cell proliferation was determined by Incucyte zoom live cell analysis system from Essen Bioscience. 2000 cells / well were seeded in a 96-well plate with $10 \%$ FBS containing media. Cells were incubated in the Incucyte zoom incubator at $37^{\circ} \mathrm{C}$ with $5 \% \mathrm{CO}_{2}$. Images were taken at every 4 hours and cell occupancy percentage over time was determined.

\section{Co-immunoprecipitation (co-IP)}

$200 \mu \mathrm{g}$ of cell lysate was incubated with TLK1 or GFP specific antibodies for 4 hours on ice. $50 \mu \mathrm{l}$ of preequilibrated protein A/G agarose beads (50\% slurry) (SCBT, Dallas, TX, USA, cat\# sc-2003) was added and incubated overnight at $4^{\circ} \mathrm{C}$ with rotation. Beads were washed three times with cell lysis buffer and bound proteins were eluted with $1 \mathrm{X}$ Laemmli buffer. The eluted sample was further subjected to WB analysis.

\section{Protein Purification}

Recombinant full length his-tagged TLK1B and his-tagged MK5 protein was purified according to the published literature $[12,40]$.

\section{His and GST-pull down assay}

Cell lysates were prepared in 1X RIPA lysis buffer (SCBT, Dallas, TX, USA, cat\# 24948). $20 \mu \mathrm{g}$ of Histagged TLK1B or GST-tagged MK5 (Sino biological, Chesterbrook, PA, USA, cat\# 13655-H20B) was added to $400 \mu \mathrm{g}$ of cell lysates and incubated on ice for 30 minutes. $30 \mu \mathrm{l}$ of equilibrated $\mathrm{Ni}^{2+}$-NTA agarose (50\% slurry) (Qiagen, Germantown, MD, USA, cat\# 30210 ) or glutathione agarose (50\% slurry) (Thermo Fisher, Waltham, MA, USA, cat\# 16100) was added to the mixture and incubated on ice for 30 minutes. The reactions were spun down and beads were washed three times with 1X RIPA lysis buffer. Bound proteins were eluted using $1 X$ Laemmli buffer and run into SDS-PAGE gel for WB analysis.

\section{ADP hunter assay}

ADP hunter assay was conducted to determine the catalytic activity of lab purified recombinant MK5 kinase using either HSP27 (Abcam, Cambridge, MA, USA, cat\# ab48740) or PRAKtide (KKLRRTLSVA) (Alan Scientific, Gaithersburg, MD, USA), as previously described [12].

\section{In vitro kinase assay}

Radioactive in vitro kinase (IVK) assay was conducted by incubating HSP27 with either TLK1B or MK5 or with both TLK1B and MK5, as previously described [12]. For mass spectrometric (MS) analysis, another IVK assay was conducted by incubating either MK5 and/or TLK1 with non-radiolabeled ATP. MK5 gel bands were excised and sent to the Kentucky MS facility.

\section{Identification of MK5 phosphorylation by mass spectrometry}


Identification of the phosphoresidues of MK5 by TLK1 was conducted at the University of Kentucky Proteomics Core Facility as previously described [12].

\section{Immunohistochemistry}

For TRAMP mice IHC staining, prostates were harvested, formalin fixed, processed, and paraffin embedded. Prostate TMA was prepared as previously described [10]. Tumor tissues were cut in serial 5 $\mu \mathrm{m}$ thin sections, deparaffinized in xylene, rehydrated with decreased concentrations of ethanol, and boiled in unmasking solution (10mM sodium citrate + 1mM EDTA) for 15 minutes for antigen retrieval. Cellular peroxidase was quenched with $3 \%$ hydrogen peroxide. Afterwards, tissue sections were blocked in 3\% BSA for 1 hour followed by the incubation of primary antibody against pMK5 Ser354 (1:200 dilutions) overnight at $4^{\circ} \mathrm{C}$. Sections were washed three times and incubated in secondary antibody (Vector Laboratories, Burlingame, CA, cat\# PK-6200) for 1 hour at room temperature. After washing, the sections were then incubated in Vectastain $A B C$ reagent (Vector Laboratories, Burlingame, CA, cat\# PK$6200)$ for 30 minutes. The sections were washed again and incubated in DAB substrates for 2 minutes (Vector Laboratories, Burlingame, CA, cat\# SK-4105). Slides were then washed, counterstained with hematoxylin, dehydrated, and cover-slipped. Imaging and quantification were done by our pathologist, Dr. Judy King.

\section{Western blotting}

Cell were harvested and lysed in 1X RIPA buffer by sonication. Protein concentration was measure using Pierce BCA protein assay kit (Thermo Scientific, Waltham, MA, USA, cat\# 23225). Samples were run in SDS-PAGE gels and transferred to a PVDF membrane. After blocking in $5 \%$ non-fat dry milk, the membranes were incubated in primary antibodies overnight at $4^{\circ} \mathrm{C}$ followed by the incubation in HRP conjugated secondary antibodies for 1 hour at room temperature. The blots were incubated in ECL substrates (Thermo Scientific, Waltham, MA, USA, cat\# 32106) and imaging was conducted using Biorad ChemiDoc imaging system (Biorad, Hercules, CA, USA, cat\# 12003154).

\section{Bioinformatics analysis}

Genomic amplification of MK5 of PCa patients were generated using cBioportal database (accessed on February 7, 2020) from the Trento/Broad/Cornell (2016), SU2C/PCF Dream Team (Cell, 2015 and PNAS, 2019), MCTP (Nature, 2012), and other datasets. mRNA expression of both TLK1 and MK5 based on regional lymph node metastases and Gleason scores from PCa TCGA datasets was generated using UALCAN online platform (accessed on February 7, 2020).

\section{Statistical analysis}

Statistical calculations were performed using GraphPad Prism 9 and Microsoft Excel software. Data quantifications are expressed as mean \pm standard error of the mean (SEM). Statistical significance was calculated by 2-tailed Student $t$ test when comparing the mean between two groups, or by one-way ANOVA followed by Tukey's posthoc analysis when comparing more than two groups. $p$-values $<0.05$ were considered significant. 


\section{Results}

\section{MK5 promotes cell motility in both non-malignant and neoplastic cell lines}

We have utilized a novel proteomic screening assay from Life Technologies to identify the interactome of TLK1B [45]. TLK1B is a spliced variant of TLK1 that lacks the first 238 amino acids in the N-terminal domain of TLK1. TLK1B is translationally regulated, shares the identical C-terminal kinase domain and is believed to have similar substrate specificity with TLK1 $[8,41]$. Briefly, biotinylated TLK1B was used to hybridize 9000 human full-length proteins spotted in duplicates on glass slides, after which, fluorophore conjugated streptavidin was used to generate the signals from the interactions between TLK1 and arrayed proteins. This assay identified 165 human proteins that interact with TLK1 with high confidence that are involved in various cellular functions such as cell cycle regulation, DNA damage repair, DNA replication, and cell motility. MK5 was identified as one of the top interactor proteins as shown in Fig. 1A where the top 20 TLK1 interactors were plotted according to their affinity p-values. Additional information of these interacting proteins including signal intensity, negative control signal (streptavidin only), Z-score, signal variance between duplicated spots, and $\mathrm{Cl}$-value (Chebyshev's Inequality $\mathrm{p}$-value) can be found in the supplementary section of Singh et al. (2017) [45]. Since MK5 promotes cellular motility in both nonmalignant (e.g., PC12 [17], HUVEC [16]) and neoplastic cells (e.g., HeLa [20]), we tested whether MK5 overexpression or depletion alters the motility rate of mouse embryonic fibroblast (MEF). MK5 was overexpressed in wild type (WT) MEF cells using eGFP-MK5 mammalian construct and exogenous MK5 level was determined by western blotting (Fig. S1A). We compared the scratch healing rate among MK5 ${ }^{-1}$ - MEF [43], WT MEF, and MK5 overexpressing MEF cells by scratch repair assay. Scratch repair appeared to be the fastest in the MK5 overexpressing MEF and slowest in the $\mathrm{MK5}^{-/-} \mathrm{MEF}$ cells (Fig. 1B and S1B).

It is noteworthy that MK5 ${ }^{-/-}$MEF cells was generated in a mixed genetic background (129/Ola X C57BL/6), whereas wild type MEF cells were isolated from C57BL/6 mice [43]. To rule out the possibility that the discrepancy in the genetic background of MK5 $5^{-/-}$MEF and WT MEF cells can affect their migration rate in scratch wound repair assay, we reconstituted MK5 in the MK5 ${ }^{-/-}$MEF cells (Fig. S2A). In addition, we tested if TLK1 alone in absence of MK5 can enhance their migration rate, by stably overexpressing TLK1 in the MK5 ${ }^{-/-}$MEF Cells (Fig. S2B). When we compared the wound healing rates among these three cell lines, namely, MK5 rescued MEF, MK5 ${ }^{-/-}$MEF, and TLK1 overexpressing $\mathrm{MK}^{-/-}$ MEF cells in a scratch repair assay, we observed that while MK5 rescued cells migrated significantly faster, there was no significant difference in the migration rate between TLK1 overexpressing $\mathrm{MK}^{-/-} \mathrm{MEF}$ and $\mathrm{MK}^{-/-}$MEF cells (Fig. 1C and S2C). The latter results indicate that without MK5, TLK1 cannot exert its function in motility promotion. To confirm our observations from the scratch assay, we utilized the 3D chemotactic trans-well migration assay in the Incucyte machine using three different extra cellular matrices (ECMs). We observed that in fibronectin, MK5 rescued cells migrated significantly faster and no difference in the migration rate of TLK1 overexpressing $\mathrm{MK5}^{-/-}$vs. MK5 ${ }^{-/-}$MEF cells (Fig. 1D and S3). Similar trends in migration were also observed using Matrigel and collagen I (Fig. S4 and S5). To examine whether reconstitution of MK5 increases the proliferation rate of the cells which might contribute to 
enhanced migration rate that we observed in the scratch and chemotactic migration assays, we conducted a proliferation assay between MEF MK5 ${ }^{-/-}$and MEF MK5 $5^{-/-}$GFP-MK5 cells. MK5 reconstitution did not significantly increase the proliferation rate compared to $\mathrm{MK}^{-/-} \mathrm{MEF}$ cells (Fig. 1E and S6). We conducted another scratch experiment with LNCaP cells, an androgen dependent PCa cell line. LNCaP cells were transfected with either eGFP-MK5 or TLK1 kinase dead (KD) dominant negative mammalian construct (Fig. S7A) [41]. We observed significantly faster wound healing in the MK5 overexpressing LNCaP cells compared to the TLK1 KD and WT LNCaP cells (Fig. 1F and S7B). Overall, these findings suggest MK5 as a promotility factor, in addition, TLK1 alone in absence of MK5 cannot increase the migration rate of the cells.

\section{TLK1 knockdown/inhibition results in reduced cell migration rate}

Emerging evidence suggest that TLK2 can promote cancer invasiveness by enhancing the migration and invasion of breast cancer and glioblastoma cell. Since TLK1 and TLK2 share high homology in the amino acid sequence and are believed to have partly redundant functions, we hypothesize that TLK1 can also regulate cellular motility. To examine whether TLK1 acts a potential factor to regulate cell motility, we took both genetic and pharmacologic approach. Both siRNA mediated knockdown and pharmacologic inhibition of TLK1 using small molecule inhibitor (J54) resulted in the reduced scratch healing rate in WT MEF cells compared to the control cells (Fig. 2A, 2B, and 2C). J54 is a potent second generation phenothiazine derivative specific for TLK1 [44]. Scratch healing rate of TLK1 depleted and TLK1 inhibited WT MEF cells was similar and there is no statistical difference between these two experimental groups. Therefore, we opted to use J54 for our next experiments. J54 treatment also significantly reduces the wound healing rate of MK5 rescued $\mathrm{MK5}^{-/-}$MEF cells compared to the vehicle (DMSO) treated MK5 rescued cells (Fig. 2D and 2E). These findings establish both TLK1 and MK5 as promotility factors and we hypothesize that MK5 may function as a downstream effector of TLK1 to promote cellular motility. Unexpectedly, TLK1 inhibition by J54 also resulted in reduced wound closure in MK5 ${ }^{-/-}$MEF cells which suggest that TLK1 may promote cellular migration through additional downstream effectors even in the absence of MK5 (Fig. S8A and S8B).

\section{TLK1 interacts with MK5 both in vitro and in cultured cells}

Results obtained from our protoarray assay demonstrated that TLK1B interacts with MK5 in vitro (Fig. 1A). To test whether TLK1 interacts with MK5 in cultured cells, we overexpressed MK5 in HEK 293 cells using eGFP-MK5 mammalian construct and conducted a co-immunoprecipitation (co-IP) experiment. Immunoprecipitations of TLK1 using TLK1 specific antibodies brought down both exogenous and endogenous MK5, which was confirmed by western blotting (Fig. 3A). Reciprocal immunoprecipitation of MK5 using GFP specific antibody also revealed the presence of TLK1 in the co-IP (Fig. 3B). These data suggest that TLK1 and MK5 form a complex in cells. We confirmed this observation performing His- and GST- pull down experiments independently. Incubation of purified recombinant his- tagged TLK1B with MK5 overexpressing HEK cell lysates precipitated both endogenous MK5 and exogenously expressed GFP-MK5, as determined by WB (Fig. 3C). Similarly, incubation of purified recombinant GST-tagged MK5 
with TLK1 \& MK5 co-expressing cell lysate precipitated both TLK1 (Fig. 3D, left panel) and GFP-MK5 (Fig. 3D, right panel). Together, these results indicate that TLK1 interacts directly with MK5. The observation that endogenous MK5 was also pulled down in both co-IP (Fig. 3B) and GST- pull down assay (Fig. 3D, right panel) suggest that endogenous MK5 may dimerize with exogenously expressed GFP-MK5 and/or GST-MK5.

\section{TLK1 Phosphorylates MK5 both in vitro and in cultured cells and increases its catalytic activity}

We examined whether TLK1 possesses the ability to phosphorylate MK5 in vitro and/or in cultured cells. We first purified recombinant his- tagged TLK1B and recombinant his-tagged MK5 following previously published protocol (Fig. 4A and 4B) [12,40]. We confirmed the catalytic activity of MK5 using MK5 specific protein and peptide substrate, HSP27 (Fig. 4C) and PRAKtide (Fig. S9), respectively by ADP hunter assay. Incubation of purified recombinant his-tagged TLK1B with both purified recombinant histagged MK5 and HSP27 in an IVK assay synergistically increases the phosphorylation level of MK5 and intrinsic catalytic activity of MK5 towards its known substrate, HSP27 (Fig. 4D and 4E). To determine whether TLK1 can phosphorylate MK5 in cultured cells, we transfected HEK 293 cells either with GFPMK5 alone or co-transfected the cells either with GFP-MK5 +TLK1 or GFP-MK5+ TLK1-kinase dead (KD) expression construct. WB analysis revealed a hyperphosphorylated form of GFP-MK5 only in MK5+ TLK1 co-expressed cell lysate that migrated slower than the main form of GFP-MK5 (Fig. 4F). Lambda protein phosphatase (LPP) treatment reduces this slower migrating band which suggest that TLK1 can phosphorylate MK5 in cultured cells. We also conducted another IVK assay by incubating both recombinant His-TLK1B and GST-MK5 in the presence of non-radioactive cold ATP. The reactions were run in an SDS-PAGE gel and the corresponding MK5 bands were excised for mass spectrometric analysis (Fig. 4G).

\section{TLK1 Phosphorylates MK5 in vitro on three unique residues which are mapped to the functional domains of MK5}

To determine the phosphopeptides of MK5 by TLK1, MK5 bands were digested by trypsin and subjected to shot-gun proteomic analysis using an LTQ-Orbitrap mass spectrometer. MS data sets are searched with MASCOT against a custom database containing human MAPKAPK5. The summary of the analysis as are follows: 1) MAP kinase-activated protein kinase 5 (MAPKAPK5 or MK5) was detected with high protein score (4686- 8482 ) and good peptides coverage (70.40-80.55\%) in both samples. 2)

Phosphorylated sites were detected in both samples. After comparing MK5 mock and MK5+TLK1B samples, a few unique phosphorylation sites were detected in MK5+TLK1B sample: S160, S348, S354, and S386 (Fig. 5 and Table S1). The constitutive phosphorylation sites present in both MK5 mock and MK5+TLK1B samples can be explained as the autophosphorylation sites of MK5 (Fig. S10). 3) S348 and S354 are within the same peptide with single phosphorylation event. After comparing the spectra, S354 was determined to be the more likely phosphorylation site. 4) Thus, we conclude that there are three unique phosphorylation sites in MK5+TLK1B sample: S160, S354, and S386. We mapped these serine residues to the domains of MK5 and identified that they are located in the kinase domain (in the 
activation loop near the residue Thr182, which needs to be phosphorylated for the catalytic activation of MK5 $[46,47])$, in the nuclear localization signal (NLS) domain, and in the ERK3 binding domain (rev. in [37]) (Fig. S11).

\section{Anti-androgen treatment increases pMK5 Ser354 level and can be inhibited by small molecule inhibitor specific to TLK1}

Anti-serum raised against one of the phosphoresidue of MK5 (Ser354), one of the sites that we identified as being target of TLK1, has recently become commercially available and we tested it to determine if this specific MK5 phosphoprotein is present in several androgen dependent and independent PCa cell lines (Fig. 6A and 6D) $[48,49]$. MK5 ${ }^{-/-}$MEF cells were used as a negative control. We also detected TLK1 in those PCa cell lines (Fig. 6A). Western blotting analysis revealed the presence of pMK5 S354 level relative to the total MK5 in all PCa cell lines tested, while no pMK5 S354 was detected in MK5 ${ }^{-/-}$MEF cells. We also previously reported that treatment of LNCaP and other androgen sensitive PCa cells with Bicalutamide results in increased expression of $\operatorname{TLK} 1 \mathrm{~B}[9,10]$. We now show that anti-androgen treatment also results in a corresponding dose-dependent increase in pMK5(S354) (Fig. 6B and 6E), suggesting that TLK1/1B may be the kinase responsible for this phosphorylation. Moreover, we treated LNCaP cells with two specific small molecule inhibitors of TLK1 (THD or J54), which resulted in the reduction of pMK5 S354 level compared to the DMSO treated control LNCaP cells, which further supports TLK1 role in MK5 Ser354 phosphorylation (Fig. 6C and 6F).

\section{Expression of pMK5-S354 in PCa progression of TRAMP mice}

We wanted to study if the presence of pMK5 presented with a pattern of progressive intensity during progression to invasive PCa in the TRAMP model, given that we have already reported the expression of TLK1 increases greatly from 12 to 30 weeks of age, particularly after castration [10]. At 12 weeks, TRAMP mice present feature of early carcinogenic progression of the prostate, with a mix of hyperproliferative lesions such as, prostatic intraepithelial neoplasia (PIN), to well differentiated adenocarcinomas, and in some case some areas of invasiveness with breakdown of the basement membrane. These features are remarkably well represented in Fig. 7A (top panels), which clearly shows that the areas of PIN, confined $\mathrm{Ca}$, and locally invasive $\mathrm{Ca}$ are stained progressively darker upon pMK5IHC, compared to normal acini (Fig. 7A and 7B). At 26 weeks the PCa has progressed to fully invasive, and the cancer cells are spread thought the glands (Fig. 7A, bottom panels).

\section{pMK5 Ser354 is elevated in patients with high grade metastatic prostate cancer}

We interrogated our institutional PCa TMA (with African American prevalence) to establish possible clinicopathologic correlations based on the Gleason score/s (GS), regional lymph node metastasis, and the levels of pMK5 Ser354. pMK5 Ser354 staining intensity was progressively higher in tumor samples with increasing Gleason scores and lymph nodes metastases. For instances, our representative IHC images revealed higher positive staining of pMK5 ser354 of several GS 8-9 tumors compared to GS 6 (Fig. 8). Similar increase was also observed in the tumors with 1-3 lymph nodes metastasis (N1). A very 
notable feature was that, regardless of GS (the key token of pathology determination to date) the nuclear to cytoplasm ratio of pMK5 was decreased in several samples, including some N1 of not the highest GS grade (Table S2). MK5 is generally localized to the nuclei, but it shuttles to the cytoplasm upon MAPK activation $[42,50]$, where it is believed to regulate actin cytoskeletal reorganization. While our TMA does not report on patients' outcome, it would be intriguing to study this in the future to establish if the pMK5 nuclear/cytoplasm ratio can be used as a prognostic indicator. Generally, tumors with GS $\leq 6$ are considered as low risk tumors with well-differentiated PCa cells, GS=7 is considered as moderate risk, and $G S \geq 8$ is considered as high risk tumors with poorly differentiated PCa cells that have higher migratory and invasive potentials. Higher phosphorylation of MK5 may suggest TLK1-MK5 signaling as a key driver of metastasis in PCa subjects.

\section{Pharmacologic inhibition of MK5 strongly reduces wound healing of both androgen dependent and}

\section{independent PCa cells}

To establish that MK5 is indeed important for cell motility, we tested a panel of PCa cell lines, including LNCaP, C4-2B, PC3, DU145, and 22RV1 with the specific MK5 inhibitor GLPG-0259 (Fig. 9, S12, and S13) using the scratch repair assay via the Incucyte. In all cell lines tested, GLPG resulted in a strong immobilization effect with no apparent toxicity in terms of loss of viable cells. This effect was noticeable within few hours after addition of the drug, with already maximal effect at $10 \mu \mathrm{M}$, which is close to the established IC $\mathrm{C}_{50}$ for GLPG0259 [51, 52]. These findings further confirm the promotility role of MK5 in PCa cells, where TLK1 interaction and phosphorylation of MK5 promotes motility.

\section{Bioinformatic analysis revealed genomic amplification and upregulation of both TLK1 and MK5 in metastatic tumors}

Previous studies reported TLK1 as one of the major drivers of CRPC progression after ADT[9, 10, 12$]$. Androgen ablation also results in the activation of some MAPK pathways which will further increase tumor aggressiveness. We interrogated publicly available databases to determine TLK1 and MK5 status in actual PCa patients with advanced tumors. Analysis of TCGA, SU2C, Broad/Cornell, and other PCa datasets revealed genomic copy number increase of MK5 in CRPC patients compared to localized tumors (Fig. 10A). We analyzed the mRNA expression of both TLK1 and MK5 of PCa tumors with different nodal metastatic status using the UALCAN online bioinformatic tool. Tumors with 1-3 regional nodal metastatic lesions (N1) showed slightly higher expression of TLK1, but significant upregulation of MK5, compared to tumors with no nodal metastatic lesion (NO) (Fig. 10B and 10C) was observed. Similarly, consistent upregulation of both TLK1 and MK5 was observed in tumors with increasing Gleason scores (Fig. 10D and 10E). These may suggest that genomic amplification or higher mRNA expression of TLK1 and MK5 may have association in increasing tumor aggressiveness and metastatic potential.

\section{Discussion}


Prostate cancer $(\mathrm{PCa})$ is a slow progressing disease where the mortality rate peaks with the metastasis of cancer cells to other vital organs. While organ confined $\mathrm{PCa}$ is more clinically manageable and has a nearly $100 \%$ five years survival rate, PCa metastasis drastically decreases the survival of the patients. The median survival rate of the prostate cancer patients with liver, lung, bone, and lymph nodes metastasis are approximately 14, 19, 21, and 32 months respectively [53]. Failure of ADT leads to more aggressive malignancy and drives metastatic progression of the PCa. However, the molecular mechanism of this metastatic dissemination is not fully understood yet. Motility and invasion are two essential cellular processes for cancer cells to get detached from the primary tumor site and disseminated throughout the body. Motility and invasion are regulated by the concerted action of several factors, coordination of multiple signal transduction pathways, gene expression, cytoskeletal changes, and remodeling of surrounding extracellular matrices. These allow the cells to invade and migrate through new tissues.

Our discovery of TLK1B-MK5 interaction through protoarray assay prompted us to investigate the role of TLK1 and MK5 in motility in both non-malignant and neoplastic cell lines as TLK1 paralog, TLK2 and MK5 were reported to increase cellular motility and invasion $[13,14,16,18,20]$. Both genetic depletion and pharmacologic inhibition of MK5 and TLK1 independently resulted in significantly reduced wound healing, whereas overexpression/rescue of MK5 enhances the wound closure rate (Fig. 1, 2, and 9). However, overexpression of TLK1 alone in MK5 depleted cells did not promote the migration, which suggests TLK1 must exert its promotility function primarily through MK5 (Fig. 1C and 1D). We further confirmed TLK1-MK5 interaction by co-IP, His and GST pull down assay and elucidated that TLK1 can phosphorylate MK5 in three serine residues (S160, S354, and S386) which are located on some functional domains of MK5 (Fig. 3-5). Thus, MK5 is a direct substrate of TLK1 which increases its intrinsic kinase activity in vitro (Fig. 4D and 4E). We reported the presence of TLK1 and MK5 phosphoprotein (pMK5 S354) in all major androgen dependent and independent PCa cell lines and demonstrated that anti-androgen (bicalutamide) treatment increases the pMK5 S354 level in a dosedependent fashion (Fig. 6A, 6B, 6D, and 6E). Pharmacologic inhibition of TLK1 reduces this pMK5 level which suggests TLK1 as an authentic pMK5 S354 kinase (6C and 6F).

In PCa, TLK1/1B-MK5 signaling axis might be crucial as the ADT related stress increases TLK1/1B level and activates compensatory MAPK signaling, which may result in the activation and stabilization of MK5 $[9,54]$. Through our bioinformatics study using TCGA and other publicly available datasets, we also observed that both TLK1 and MK5 are upregulated in metastatic and advanced PCa (Fig. 10). Cellular abundance of these kinases may increase their interaction frequencies and start a novel signaling transduction pathway to promote cellular migration. When the tumor grows sufficiently large, cells may shed from the primary tumor site and find a secondary location to avoid hypoxic stress. We propose that TLK1-MK5 signaling aids this process by increasing the motility and invasion of the cells. Interrogation of a PCa TMA as well as IHC of TRAMP mice of different stages of PCa progression leading to metastatic spread is supportive of the notion that pMK5 S354 is potential marker of disease progression and in particular of invasive PCa (Figs. 7 and 8). Notably to date, Pathologists mainly use H\&E staining in the attempt to determine PCa features that predict worse outcome. However, a relatively mild GS is often no 
predictor of better outcome, particularly if accompanied by known presence of nodal involvement (N1). A reliable IHC marker, with an established cellular function, that better predicts the intrinsic invasive potential of the PCa cells would be a significant addition to available tools for Pathology determinations.

Mechanistically, Cellular motility is orchestrated by the formation of protruding bodies (lamellipodia, filopodia, invadopodia) which are rich in sub-membranous actin filaments. MK5 can regulate actin cytoskeletal reorganization through its bona fide substrate HSP27. Non-phosphorylated HSP27 functions as a capping protein to inhibit F-actin polymerization and thus inhibits stress fiber formation [55-59]. Upon external stress related stimuli, activated MK5 can phosphorylate HSP27 on Ser15, Ser78, and Ser82 residues, which hinders its capping activity and allows monomeric actin to grow into filamentous actin $[17,18,20,46,60,61]$. Ugo Moens group demonstrated that catalytic subunit of PKA can interact and phosphorylate MK5 on Ser115 residue which induces its nuclear export and F-actin polymerization [17, 18, 62]. Similarly, TLK1 interaction and phosphorylation of MK5 may also promote cellular motility by actin remodeling mediated through HSP27 phosphorylation (Fig. 11). In fact, our IVK analysis shows that TLK1 increases MK5 catalytic activity towards HSP27 phosphorylation (Fig. 4D and 4E).

Another potential mechanism could be focal adhesion complex modifications by TLK1-MK5 signaling that promotes cell motility. Since both TLKs and MK5 are known to form complexes with FAK [16, 22], Src $[13,14,22]$, and paxillin [22], it is likely that these complexes depend on TLK1-MK5 interaction. TLK2 is reported to complex with Src and activate EGFR/Src/FAK signaling pathway to promote the migration and invasion in breast adenocarcinoma and glioblastoma cells $[13,14]$. Yoshizuka et al. demonstrated that MK5 increases motility in endothelial cells by phosphorylating FAK on Tyr397 residue and localizes it to focal adhesions in advanced skin carcinogenesis [16]. Additionally, another group reported that recombinant MK5 can phosphorylate FAK, Src, and paxillin in IVK assays and form complexes with FAK and Src and that endogenous MK5 localizes to focal adhesions [22]. We suggest that TLK1 interacts with MK5 and recruits it to focal adhesions where both TLK1 and MK5 may form complex with either FAK or Src or Paxillin, phosphorylate and activate them and transduce downstream signals (Fig. 11). FAK activation by TLK1-MK5 axis can regulate the affinity and avidity of integrins towards ECM as well as initiate a feed forward loop of MK5 activation through Rac1 > PAK > ERK3/4 > MK5 signaling [63-66].

MK5 can be activated by both typical and atypical MAPKs, p38 [46] and ERK3/4 [67-69], respectively. MK5 full activation needs the phosphorylation of Thr182 residue, which is located in its activation loop (Fig. S11) [46, 68]. One of the phosphorylations of MK5 by TLK1 is mapped to Ser160 and this site is located on the catalytic domain near to the activation loop of MK5. This phosphorylation may cause a conformational change of MK5 and increase its affinity towards its substrates. On the other hand, TLK1 phosphorylation of MK5 may also enhance nucleocytoplasmic localization of MK5, while MK5 is predominantly nuclear in the resting cells. This is, in agreement with the findings of others that phosphorylation of MK5 by its interaction partners causes the redistribution of MK5 from nucleus to cytoplasm [42, 50, 70-72]. TLK1 mediated phosphorylation on Ser354 residue, which lies on the NLS domain of MK5, may mask the NLS and promote its nuclear export to exert its functions on motility enhancement. 


\section{Conclusion}

Implementation of ADT and novel antiandrogens has made an impact in the early survival of PCa patients, but presence of metastasis remains a significant poor prognostic indicator. Inhibition of metastatic dissemination seems to be the first and foremost challenge in the treatment of mCRPC patients. We identified a novel signaling axis (TLK1-MK5) which is active in majority of the PCa cell lines and demonstrated that disruption of this signaling significantly reduces PCa cell migration. Further research is needed to determine whether the inhibition of TLK1-MK5 signaling can reduce PCa metastasis in xenograft mice model using TLK1 and/or MK5 inhibitor. Successful completion of such work may result in the application of these inhibitors in combination with other treatment strategies to delay PCa metastatic burden and lethal progression.

\section{Abbreviations}

ADT

Androgen deprivation therapy

Co-IP

Co-immunoprecipitation

CRPC

Castration resistant prostate cancer

ECM

Extra cellular matrix

GS

Gleason score

$\mathrm{IHC}$

Immunohistochemistry

IVK

In vitro kinase

LPP

Lambda protein phosphatase

mCRPC

Metastatic castration-resistant prostate cancer

MK5

MAPK-activated protein kinase 5

MR

Mean rate

MS

Mass spectrometry

NEK1

NIMA-related kinase 1 
NLS

Nuclear localization signal

$\mathrm{PCa}$

Prostate cancer

PIN

Prostatic intraepithelial neoplasia

PRAK

p38 regulated and activated kinase

SEM

Standard error of the mean

THD

Thioridazine

TLK1

Tousled-like kinase 1

TMA

Tissue microarray

TSL

Tousled kinase

uORF

Upstream open reading frame

VC

Vehicle control

\section{Declarations}

Ethics approval and consent to participate: Construction of the PCa TMA was made at LSUHSC following IRB approval and consented donors. These were deidentified for this study following a second IRB approval. Animal studies were approved by our IACUC.

Consent for publication: N/A

Availability of supporting data: All data generated or analyzed during this study and its supplementary information files are included in this published article. Cell lines and most reagents generated for this study are available upon request.

Competing Interests: The authors declare that no competing interests exist.

Funding: This work was supported by DoD-PCRP grant W81XWH-17-1-0417 to ADB

Authors' contributions: MIK and VS generated most of the data and MIK designed some of the research. JK provided expertise and analysis of the TMA and mouse IHC studies. MIK wrote much of the paper and 
conducted the analysis of the TCGA data. ADB directed and designed the research and wrote portions of the paper.

Acknowledgements: We like to thank Dr. Erik First (LSUHSC- Shreveport) for his assistance with MK5 protein purification. We like to thank the INLET facility of LSUHSC- Shreveport, especially, Dr. Ana maria Dragoi, Dr. Jennifer Carroll-Duhon, and Brian Latimer for their assistance in working with the Incucyte machines. We also like to thank Dr. Jing Chen and Dr. Haining Zhu from University of Kentucky Proteomics Core Facility for conducting the mass spectrometric analysis.

Authors' information: N/A

\section{References}

1. Logothetis CJ, Lin S-H. Osteoblasts in prostate cancer metastasis to bone. Nat Rev Cancer. 2005;5(1):21-8.

2. Tarver T: Cancer facts \& Figs. 2012. American cancer society (ACS) Atlanta, GA: American Cancer Society, 2012. 66 p., pdf. Available from. In.: Taylor \& Francis; 2012.

3. Deng X, He G, Liu J, Luo F, Peng X, Tang S, Gao Z, Lin Q, Keller JM, Yang T. Recent advances in bonetargeted therapies of metastatic prostate cancer. Cancer treatment reviews. 2014;40(6):730-8.

4. Roe J, Rivin C, Sessions R, Feldmann K, Zambryski P. The Tousled gene in A. thaliana encodes a protein kinase homolog that is required for leaf and flower development. Cell. 1993;75(5):939-50.

5. Roe J, Rivin C, Sessions R, Feldmann K, Zambryski P, Nemhauser J, Zambryski P. TOUSLED Participates in Apical Tissue Formation during Gynoecium Development in Arabidopsis. PLANT CELL. 1997;9(3):335-53.

6. Sillje HH, Takahashi K, Tanaka K, Van Houwe G, Nigg EA. Mammalian homologues of the plant Tousled gene code for cell-cycle-regulated kinases with maximal activities linked to ongoing DNA replication. Embo J. 1999;18(20):5691-702.

7. Segura-Bayona S, Stracker TH: The Tousled-like kinases regulate genome and epigenome stability: implications in development and disease. Cell Mol Life Sci 2019, 76(19):3827-3841. doi: 3810.1007/s00018-00019-03208-z. Epub 02019 Jul 00013.

8. Li Y, DeFatta R, Anthony C, Sunavala G, De Benedetti A. A translationally regulated Tousled kinase phosphorylates histone $\mathrm{H} 3$ and confers radioresistance when overexpressed. Oncogene. 2001;20(6):726-38.

9. Singh V, Jaiswal P, Ghosh I, Koul HK, Yu X, De Benedetti A. Targeting the TLK1/NEK1 DDR axis with Thioridazine suppresses outgrowth of Androgen Independent Prostate tumors. International journal of cancer. 2019;145:1055-67.

10. Singh V, Jaiswal PK, Ghosh I, Koul HK, Yu X, De Benedetti A: The TLK1-Nek1 axis promotes prostate cancer progression. Cancer Lett 2019, 453:131-141. DOI 110.1016/j.canlet.2019.1003.1041. Epub 2019 Mar 1027. 
11. Singh V, Khalil MI, De Benedetti A. The TLK1/Nek1 axis contributes to mitochondrial integrity and apoptosis prevention via phosphorylation of VDAC1. Cell Cycle. 2020;9:1-13.

12. Khalil MI, Ghosh I, Singh V, Chen J, Zhu H, De Benedetti A. NEK1 Phosphorylation of YAP Promotes Its Stabilization and Transcriptional Output. Cancers. 2020;12(12):3666.

13. Kim JA, Tan Y, Wang X, Cao X, Veeraraghavan J, Liang Y, Edwards DP, Huang S, Pan X, Li K, et al: Comprehensive functional analysis of the tousled-like kinase 2 frequently amplified in aggressive luminal breast cancers. Nat Commun 2016, 7:12991.(doi):10.1038/ncomms12991.

14. Lin M, Yao Z, Zhao N, Zhang C. TLK2 enhances aggressive phenotypes of glioblastoma cells through the activation of SRC signaling pathway. Cancer Biol Ther. 2019;20(1):101-8.

15. Xiang W, Zhang D, Montell DJ. Tousled-like kinase regulates cytokine-mediated communication between cooperating cell types during collective border cell migration. Mol Biol Cell. 2016;27(1):129.

16. Yoshizuka N, Chen RM, Xu Z, Liao R, Hong L, Hu WY, Yu G, Han J, Chen L, Sun P. A novel function of p38-regulated/activated kinase in endothelial cell migration and tumor angiogenesis. Mol Cell Biol. 2012;32(3):606-18.

17. Gerits N, Mikalsen T, Kostenko S, Shiryaev A, Johannessen M, Moens U. Modulation of F-actin rearrangement by the cyclic AMP/cAMP-dependent protein kinase (PKA) pathway is mediated by MAPK-activated protein kinase 5 and requires PKA-induced nuclear export of MK5. J Biol Chem. 2007;282(51):37232-43.

18. Kostenko S, Johannessen M, Moens U. PKA-induced F-actin rearrangement requires phosphorylation of Hsp27 by the MAPKAP kinase MK5. Cell Signal. 2009;21(5):712-8.

19. Nawaito SA, Sahadevan P, Clavet-Lanthier ME, Pouliot P, Sahmi F, Shi Y, Gillis MA, Lesage F, Gaestel $M$, Sirois MG, et al. MK5 haplodeficiency decreases collagen deposition and scar size during postmyocardial infarction wound repair. Am J Physiol Heart Circ Physiol. 2019;316(6):H1281-96.

20. Tak H, Jang E, Kim SB, Park J, Suk J, Yoon YS, Ahn JK, Lee JH, Joe CO. 14-3-3epsilon inhibits MK5mediated cell migration by disrupting F-actin polymerization. Cell Signal. 2007;19(11):2379-87.

21. Al-Mahdi R, Babteen N, Thillai K, Holt M, Johansen B, Wetting HL, Seternes OM, Wells CM. A novel role for atypical MAPK kinase ERK3 in regulating breast cancer cell morphology and migration. Cell Adh Migr. 2015;9(6):483-94.

22. Dwyer SF, Gelman IH. Cross-phosphorylation and interaction between Src/FAK and MAPKAP5/PRAK in early focal adhesions controls cell motility. Journal of cancer biology \& research 2014, 2(1).

23. Stohr N, Kohn M, Lederer M, Glass M, Reinke C, Singer RH, Huttelmaier S. IGF2BP1 promotes cell migration by regulating MK5 and PTEN signaling. Genes Dev. 2012;26(2):176-89.

24. Kress TR, Cannell IG, Brenkman AB, Samans B, Gaestel M, Roepman P, Burgering BM, Bushell M, Rosenwald A, Eilers M. The MK5/PRAK kinase and Myc form a negative feedback loop that is disrupted during colorectal tumorigenesis. Mol Cell. 2011;41(4):445-57.

25. Sun P, Yoshizuka N, New L, Moser BA, Li Y, Liao R, Xie C, Chen J, Deng Q, Yamout M, et al. PRAK is essential for ras-induced senescence and tumor suppression. Cell. 2007;128(2):295-308. 
26. Perander M, Keyse SM, Seternes O-M. Does MK5 reconcile classical and atypical MAP kinases. Front Biosci. 2008;13(4617):24.

27. Sahadevan P, Allen BG. MK5: A novel regulator of cardiac fibroblast function? IUBMB Life. 2017;69(10):785-94.

28. Kostenko S, Jensen KL, Moens U. Phosphorylation of heat shock protein 40 (Hsp40/DnaJB1) by mitogen-activated protein kinase-activated protein kinase 5 (MK5/PRAK). Int J Biochem Cell Biol. 2014;47:29-37.

29. Shiryaev A, Kostenko S, Dumitriu G, Moens U. Septin 8 is an interaction partner and in vitro substrate of MK5. World J Biol Chem. 2012;3(5):98-109.

30. Zheng M, Wang YH, Wu XN, Wu SQ, Lu BJ, Dong MQ, Zhang H, Sun P, Lin SC, Guan KL, et al. Inactivation of Rheb by PRAK-mediated phosphorylation is essential for energy-depletion-induced suppression of mTORC1. Nat Cell Biol. 2011;13(3):263-72.

31. Brand F, Schumacher S, Kant S, Menon MB, Simon R, Turgeon B, Britsch S, Meloche S, Gaestel M, Kotlyarov A. The extracellular signal-regulated kinase 3 (mitogen-activated protein kinase 6 [MAPK6])-MAPK-activated protein kinase 5 signaling complex regulates septin function and dendrite morphology. Mol Cell Biol. 2012;32(13):2467-78.

32. Nawaito SA, Sahadevan P, Sahmi F, Gaestel M, Calderone A, Allen BG. Transcript levels for extracellular matrix proteins are altered in MK5-deficient cardiac ventricular fibroblasts. J Mol Cell Cardiol. 2019;132:164-77.

33. Perander M, Keyse SM, Seternes O-M: New insights into the activation, interaction partners and possible functions of MK5/PRAK. cell 2016, 12:13.

34. Gaestel M. MAPKAP kinases - MKs - two's company, three's a crowd. Nat Rev Mol Cell Biol. 2006;7(2):120-30.

35. Gaestel M. MAPK-Activated Protein Kinases (MKs): Novel Insights and Challenges. Front Cell Dev Biol. 2015;3:88.

36. Kostenko S, Dumitriu G, Moens U. Tumour promoting and suppressing roles of the atypical MAP kinase signalling pathway ERK3/4-MK5. J Mol Signal. 2012;7(1):9.

37. Moens U, Kostenko S. Structure and function of MK5/PRAK: the loner among the mitogen-activated protein kinase-activated protein kinases. Biol Chem. 2013;394(9):1115-32.

38. Zhou J, Wan B, Liu XM, Li R, Wang Y, Yu L. MK5 is degraded in response to doxorubicin and negatively regulates doxorubicin-induced apoptosis in hepatocellular carcinoma cells. Biochem Biophys Res Commun. 2012;427(3):581-6.

39. Seo J, Kim MH, Hong H, Cho H, Park S, Kim SK, Kim J: MK5 Regulates YAP Stability and Is a Molecular Target in YAP-Driven Cancers. Cancer Res 2019, 79(24):6139-6152. doi: 6110.1158/00085472.CAN-6119-1339. Epub 2019 Oct 6132.

40. Bhoir S, Shaik A, Thiruvenkatam V, Kirubakaran S: High yield bacterial expression, purification and characterisation of bioactive Human Tousled-like Kinase 1B involved in cancer. Sci Rep 2018, 8(1):4796. doi: 4710.1038/s41598-41018-22744-41595. 
41. Sunavala-Dossabhoy G, Li Y, Williams B, De Benedetti A. A dominant negative mutant of TLK1 causes chromosome missegregation and aneuploidy in normal breast epithelial cells. BMC Cell Biol. 2003;4:16.

42. Seternes OM, Johansen B, Hegge B, Johannessen M, Keyse SM, Moens U. Both binding and activation of p38 mitogen-activated protein kinase (MAPK) play essential roles in regulation of the nucleocytoplasmic distribution of MAPK-activated protein kinase 5 by cellular stress. Mol Cell Biol. 2002;22(20):6931-45.

43. Shi Y, Kotlyarov A, Laabeta K, Gruber AD, Butt E, Marcus K, Meyer HE, Friedrich A, Volk HD, Gaestel M. Elimination of protein kinase MK5/PRAK activity by targeted homologous recombination. Mol Cell Biol. 2003;23(21):7732-41.

44. Singh V, Bhoir S, Chikhale R, Hussain J, Dwyer D, Bryce R, Kirubakaran S, De Benedetti A: Generation of Phenothiazine with Potent Anti-TLK1 Activity for Prostate Cancer Therapy. iScience 2020, 23(9).

45. Singh V, Connelly ZM, Shen X, De Benedetti A: Identification of the proteome complement of humanTLK1 reveals it binds and phosphorylates NEK1 regulating its activity. Cell Cycle 2017, 16(10):915-926. doi: 910.1080/15384101.15382017.11314421. Epub 15382017 Apr 15384120.

46. New L, Jiang Y, Zhao M, Liu K, Zhu W, Flood LJ, Kato Y, Parry GC, Han J. PRAK, a novel protein kinase regulated by the p38 MAP kinase. Embo J. 1998;17(12):3372-84.

47. Ni H, Wang XS, Diener K, Yao Z. MAPKAPK5, a novel mitogen-activated protein kinase (MAPK)activated protein kinase, is a substrate of the extracellular-regulated kinase (ERK) and p38 kinase. Biochem Biophys Res Commun. 1998;243(2):492-6.

48. Khalil MI, Singh V, Benedetti AD. The Implications of TLK1-MK5 Signaling Axis in Prostate Cancer Cell Motility and Invasion. FASEB J. 2020;34(S1):1-1.

49. Khalil MI, Singh V, De Benedetti A: TLK1-MK5 signaling axis contributes to prostate cancer cell motility and invasion. In.: AACR; 2020.

50. Aberg E, Perander M, Johansen B, Julien C, Meloche S, Keyse SM, Seternes OM. Regulation of MAPKactivated protein kinase 5 activity and subcellular localization by the atypical MAPK ERK4/MAPK4. J Biol Chem. 2006;281(46):35499-510.

51. Namour F, Vanhoutte FP, Beetens J, Blockhuys S, De Weer M, Wigerinck P: Pharmacokinetics, safety, and tolerability of GLPG0259, a mitogen-activated protein kinase-activated protein kinase 5 (MAPKAPK5) inhibitor, given as single and multiple doses to healthy male subjects. Drugs in R\&D 2012, 12(3):141-163.

52. Westhovens R, De Keyser F, Rekalov D, Nasonov EL, Beetens J, Van der Aa A, Wigerinck P, Namour F, Vanhoutte F, Durez P. Oral administration of GLPG0259, an inhibitor of MAPKAPK5, a new target for the treatment of rheumatoid arthritis: a phase II, randomised, double-blind, placebo-controlled, multicentre trial. Ann Rheum Dis. 2013;72(5):741-4.

53. Halabi S, Kelly WK, Ma H, Zhou H, Solomon NC, Fizazi K, Tangen CM, Rosenthal M, Petrylak DP, Hussain M, et al. Meta-Analysis Evaluating the Impact of Site of Metastasis on Overall Survival in Men With Castration-Resistant Prostate Cancer. J Clin Oncol. 2016;34(14):1652-9. 
54. Li Z, Sun C, Tao S, Osunkoya AO, Arnold RS, Petros JA, Zu X, Moreno CS. The JNK inhibitor AS602801 Synergizes with Enzalutamide to Kill Prostate Cancer Cells In Vitro and In Vivo and Inhibit Androgen Receptor Expression. Transl Oncol. 2020;13(4):100751.

55. Lavoie JN, Hickey E, Weber LA, Landry J. Modulation of actin microfilament dynamics and fluid phase pinocytosis by phosphorylation of heat shock protein 27. J Biol Chem. 1993;268(32):242104.

56. Huot J, Houle F, Spitz DR, Landry J. HSP27 phosphorylation-mediated resistance against actin fragmentation and cell death induced by oxidative stress. Cancer Res. 1996;56(2):273-9.

57. Song IS, Kang SS, Kim ES, Park HM, Choi CY, Tchah H, Kim JY. Heat shock protein 27 phosphorylation is involved in epithelial cell apoptosis as well as epithelial migration during corneal epithelial wound healing. Exp Eye Res. 2014;118:36-41.

58. Huot J, Lambert H, Lavoie JN, Guimond A, Houle F, Landry J: Characterization of 45-kDa/54-kDa HSP27 kinase, a stress-sensitive kinase which may activate the phosphorylation-dependent protective function of mammalian 27-kDa heat-shock protein HSP27. European journal of biochemistry 1995, 227(1-2):416-427.

59. Katsogiannou M, Andrieu C, Rocchi P. Heat shock protein 27 phosphorylation state is associated with cancer progression. Front Genet. 2014;5:346.

60. Kostenko S, Moens U. Heat shock protein 27 phosphorylation: kinases, phosphatases, functions and pathology. Cell Mol Life Sci. 2009;66(20):3289-307.

61. Shiryaev A, Dumitriu G, Moens U. Distinct roles of MK2 and MK5 in cAMP/PKA- and stress/p38MAPK-induced heat shock protein 27 phosphorylation. J Mol Signal. 2011;6(1):4.

62. Kostenko S, Shiryaev A, Gerits N, Dumitriu G, Klenow H, Johannessen M, Moens U. Serine residue 115 of MAPK-activated protein kinase MK5 is crucial for its PKA-regulated nuclear export and biological function. Cell Mol Life Sci. 2011;68(5):847-62.

63. Manser E, Leung T, Salihuddin H, Zhao Z-s, Lim L. A brain serine/threonine protein kinase activated by Cdc42 and Rac1. Nature. 1994;367(6458):40-6.

64. Rane CK, Minden A. P21 activated kinases: structure, regulation, and functions. Small GTPases. 2014;5(1):e28003.

65. De La Mota-Peynado A, Chernoff J, Beeser A. Identification of the atypical MAPK Erk3 as a novel substrate for p21-activated kinase (Pak) activity. J Biol Chem. 2011;286(15):13603-11.

66. Déléris P, Trost M, Topisirovic I, Tanguay P-L, Borden KL, Thibault P, Meloche S. Activation loop phosphorylation of ERK3/ERK4 by group I p21-activated kinases (PAKs) defines a novel PAK-ERK3/4MAPK-activated protein kinase 5 signaling pathway. J Biol Chem. 2011;286(8):6470-8.

67. Kant S, Schumacher S, Singh MK, Kispert A, Kotlyarov A, Gaestel M. Characterization of the atypical MAPK ERK4 and its activation of the MAPK-activated protein kinase MK5. J Biol Chem. 2006;281(46):35511-9.

68. Deleris P, Rousseau J, Coulombe P, Rodier G, Tanguay PL, Meloche S. Activation loop phosphorylation of the atypical MAP kinases ERK3 and ERK4 is required for binding, activation and 
cytoplasmic relocalization of MK5. J Cell Physiol. 2008;217(3):778-88.

69. Seternes OM, Mikalsen T, Johansen B, Michaelsen E, Armstrong CG, Morrice NA, Turgeon B, Meloche S, Moens U, Keyse SM. Activation of MK5/PRAK by the atypical MAP kinase ERK3 defines a novel signal transduction pathway. Embo J. 2004;23(24):4780-91.

70. Schumacher S, Laass K, Kant S, Shi Y, Visel A, Gruber AD, Kotlyarov A, Gaestel M. Scaffolding by ERK3 regulates MK5 in development. Embo J. 2004;23(24):4770-9.

71. New L, Jiang Y, Han J. Regulation of PRAK subcellular location by p38 MAP kinases. Mol Biol Cell. 2003;14(6):2603-16.

72. Li Q, Zhang N, Zhang D, Wang Y, Lin T, Wang Y, Zhou H, Ye Z, Zhang F, Lin SC, et al. Determinants that control the distinct subcellular localization of p38alpha-PRAK and p38beta-PRAK complexes. J Biol Chem. 2008;283(16):11014-23.

\section{Figures}
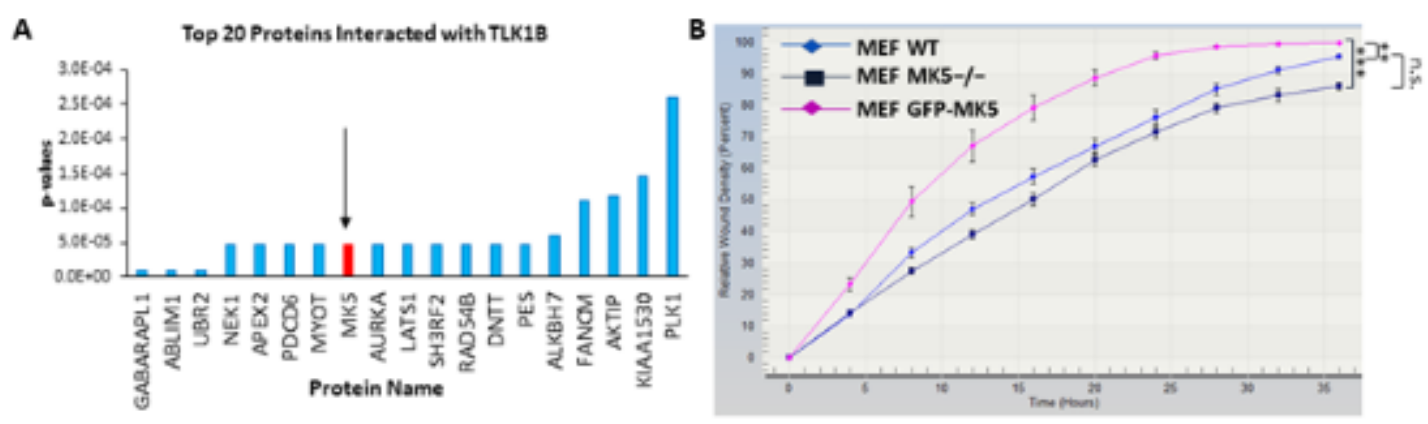

C

D
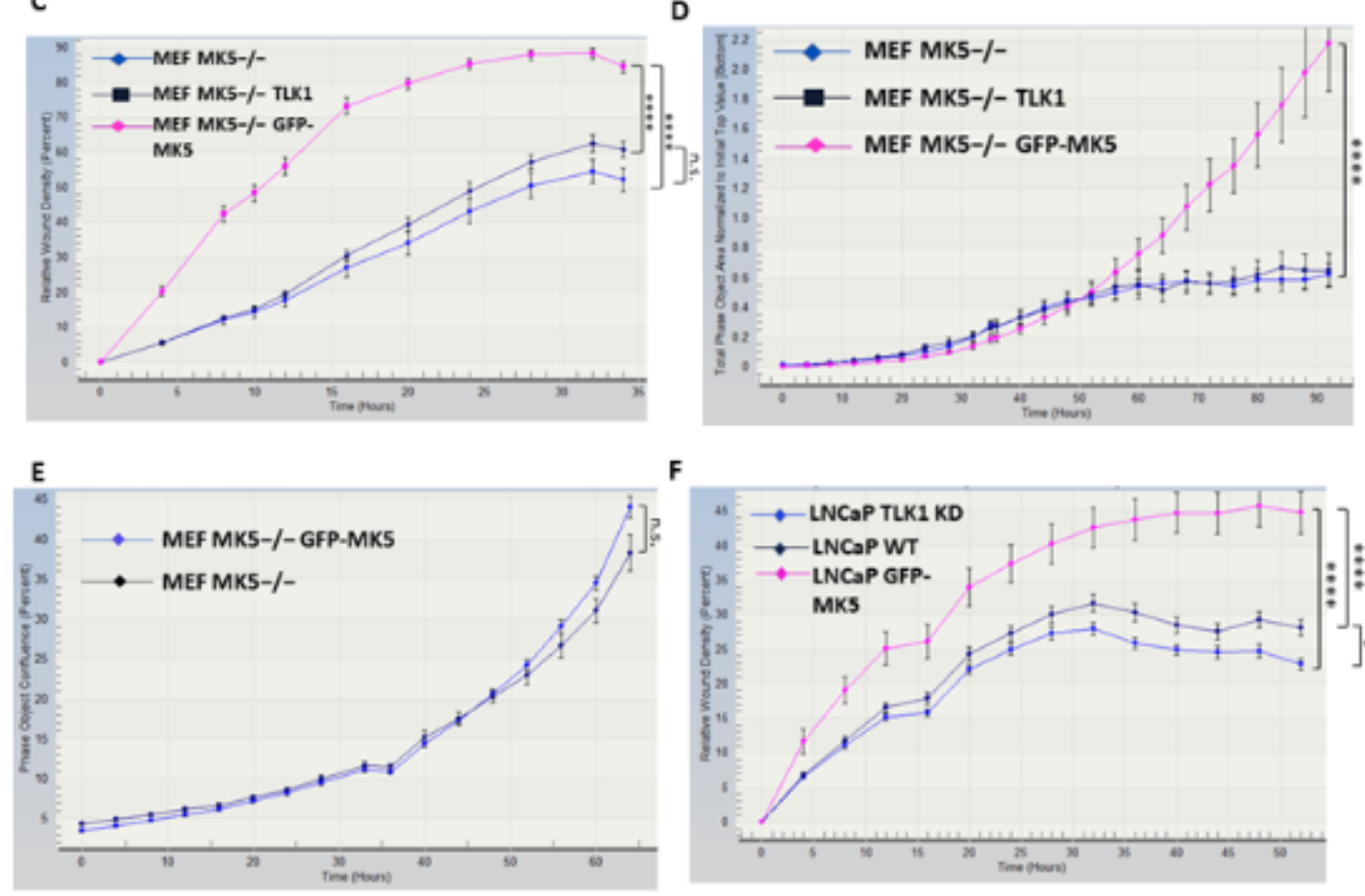

$\mathbf{F}$

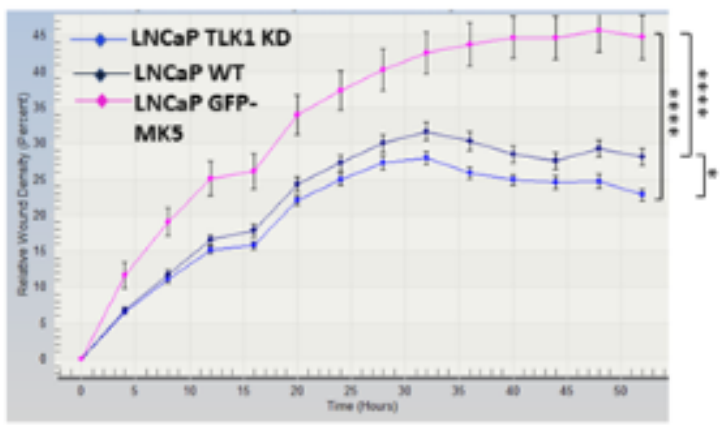


MK5 overexpression/rescue increases the migration rate of the cells, but TLK1 overexpression alone in absence of MK5 does not. A) Identification of TLK1B interactomes using protoarray assay. Top 20 proteins that interacts with TLK1B are plotted against the p-values of interaction. Interaction affinity is inversely correlated with the p-values. B, C, and F) Scratch wound repair assay was conducted to determine the 2D-migration rate by plotting relative wound density against different time points- $B$ ) Wild type MEF (MEF WT), mean rate (MR): 10.97 \pm 0.45 ; MEF MK5-/-, MR: 9.40 0.35 ; and MEF GFP-MK5, MR: 15.32 \pm 1.2. C) MEF MK5-/-, MR: 4.23 \pm 0.30 ; MEF MK5-/- TLK1, MR: 5.19 \pm 0.22 ; and MEF MK5-/- GFPMK5, MR: 13.69 \pm 0.57 . F) LNCaP WT, MR:3.97 \pm 0.10 ; LNCaP TLK1 kinase dead (KD), MR:3.47 \pm 0.11 ; and LNCaP GFP-MK5, MR: 5.10 \pm 0.16 . D) 3D chemotactic trans-well migration assay among MEF MK5-/-, MEF MK5-/- TLK1 and MEF MK5-/- GFP-MK5 cells at different time points using fibronectin coating. Migration rate was determined by plotting total object phase area (normalized to initial top value, bottom) against time among MEF MK5-/-, MR: 0.04 \pm 0.005 ; MEF MK5-/- TLK1, MR: 0.07 \pm 0.014 ; and MEF MK5-/- GFP-MK5, MR: 0.16 \pm 0.009 . E) Proliferation assay between MEF MK5-/- and MEF MK5-/- GFPMK5 cells by plotting confluence percentage over time. MEF MK5-/-, MR: 1.93 \pm 0.08 ; MEF MK5-/- GFP-

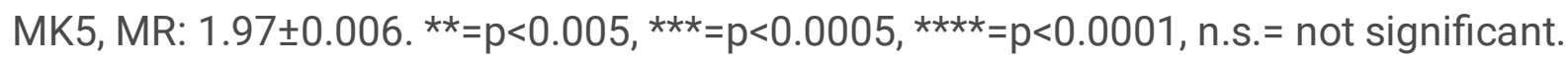
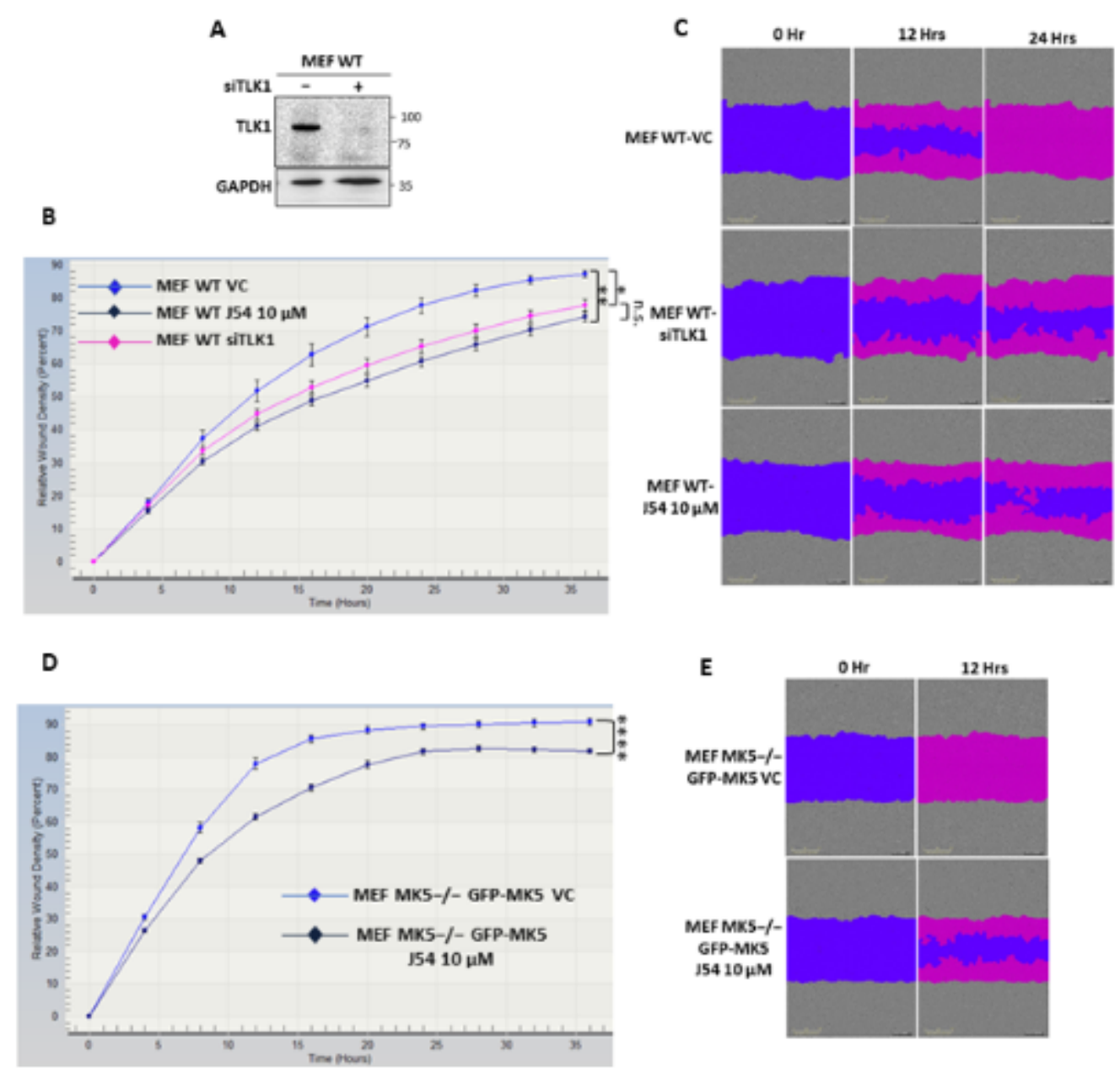

\section{Figure 2}


TLK1 knockdown/inhibition reduces cell motility rate. A) TLK1 was knockdown in wild type MEF cells using with siRNA and TLK1 level was determined by WB. GAPDH was used as a loading control. B, D) Scratch wound repair assay was conducted to determine the 2D-migration rate by plotting relative wound density against different time points in- B) MEF wild type vehicle control (MEF WT VC, MR: 12.18 \pm 0.71 ,

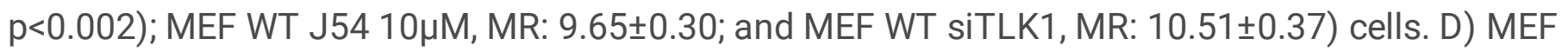

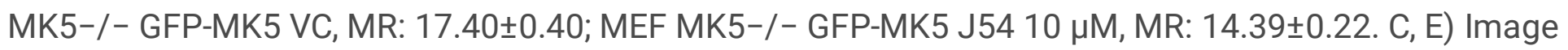
representation of the wound healing in scratch wound repair assay at different time points among $C$ ) MEF WT VC, MEF WT J54 10 $\mathrm{MM}$, and MEF WT siTLK1; and D) MEF MK5-/- GFP-MK5 VC and MEF

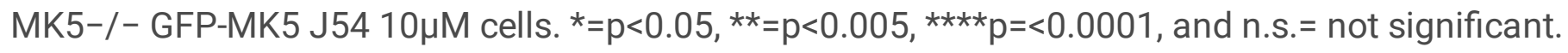
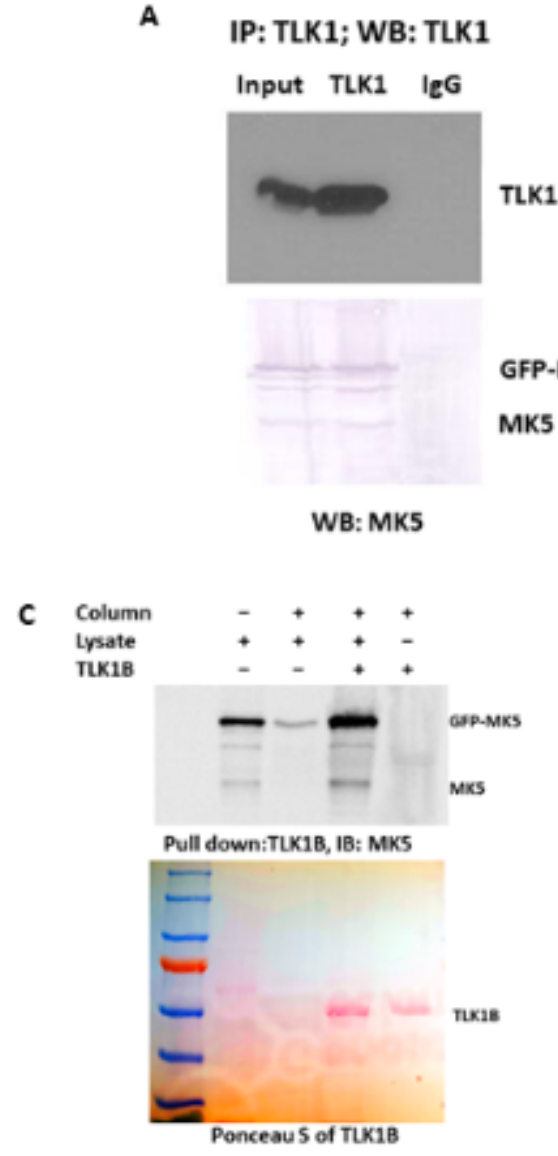

B IP: GFP; WB: MK5

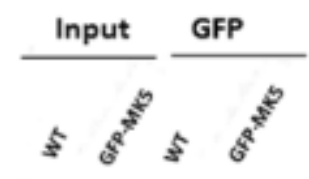

GFP-MK5

MK5

TLK1

WB: TLK1

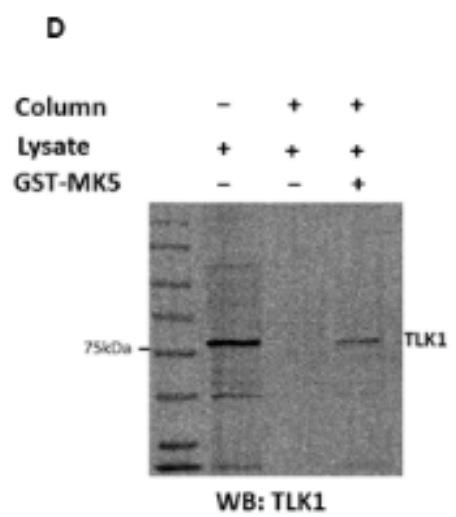

WB: TLK1

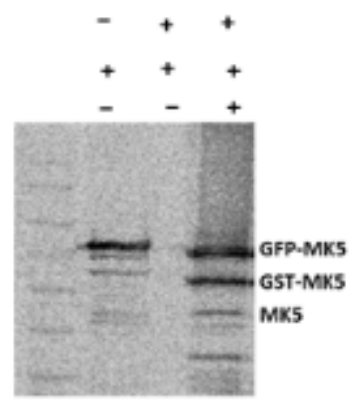

WB: MKS

\section{Figure 3}

TLK1 interacts with MK5 in cultured cells. A) Co-immunoprecipitation of TLK1 using GFP-MK5 overexpressing HEK 293 cell lysate. TLK1 was immunoprecipitated using specific antibody and blotted for both MK5 (lower panel) and TLK1 (upper panel). B) MK5 was immunoprecipitated using GFP specific antibody and blotted for both MK5 (upper panel) and TLK1 (lower panel). C) His-pull down assay using purified his-tagged TLK1B incubated with GFP-MK5 overexpressing HEK 293 cell lysate. Upper panel, WB showing both GFP- MK5 and endogenous MK5 level. Lower panel, ponceau S image showing TLK1B band. D) GST-pull down assay using purified GST-tagged MK5 incubated with GFP-MK5 and TLK1 co- 
expressing HEK 293 cell lysates. Left panel, WB detection of TLK1. Right panel, WB detection of both GFP-MK5 and GST-MK5.

A
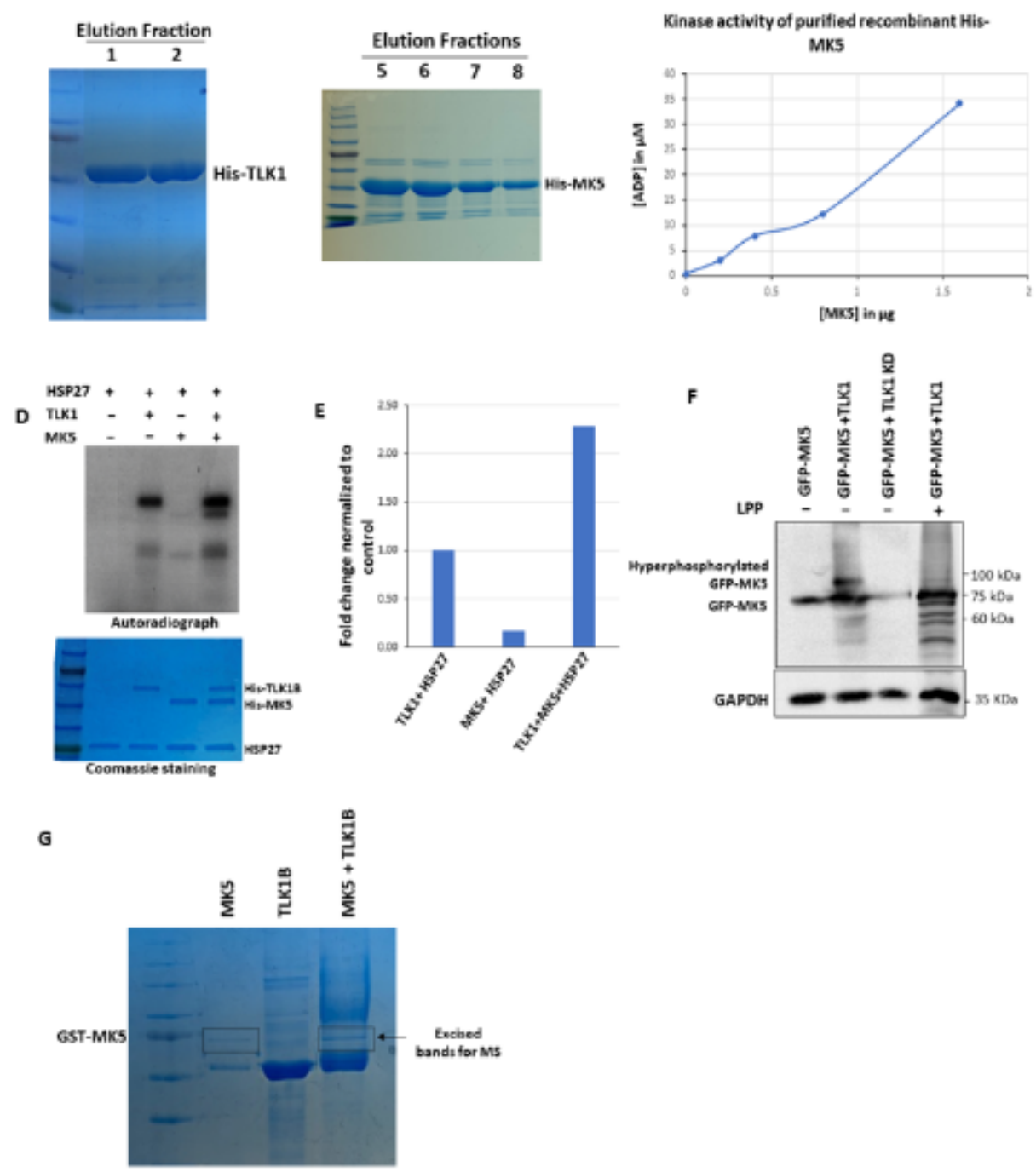

\section{Figure 4}

TLK1 phosphorylates MK5 both in vitro and in cultured cells and increases its catalytic activity. A) Purification of recombinant his-tagged TLK1B expressed in Rosetta 2 DE3 E. coli strain. Different elution fractions are shown in here. B) Purification of recombinant his-tagged MK5 expressed in Rosetta 2 DE3 E. coli strain. Different elution fractions are shown in here. C) Determination of the kinase activity of lab purified his-MK5 recombinant kinase by ADP hunter assay using protein substrate, HSP27. D) An in vitro kinase (IVK) assay using recombinant his-tagged HSP27 incubated with either his-tagged TLK1B or histagged MK5 or altogether. [ख-32P] ATP was used as a radioactive source. Top panel, an autoradiograph showing the intensity of the exposed bands of the corresponding protein. Bottom panel, Coomassie stained gel showing equal amount of protein loading. E) Relative densitometry of the fold change of HSP27 phosphorylation. F) HEK 293 cells transfected either with GFP-MK5 alone, or GFP-MK5+TLK1, or GFP-MK5+TLK1 KD. Lambda protein phosphatase (LPP) treatment was done in the 4th lane. WB showing hyperphosphorylated GFP-MK5 band in the 2 nd lane which is reduced by LPP treatment (4th lane). 
GAPDH was used as a loading control. G) IVK assay incubating either TLK1B and/or MK5 using cold ATP. Black boxed bands were excised and sent out for mass spectrometric analysis.

A

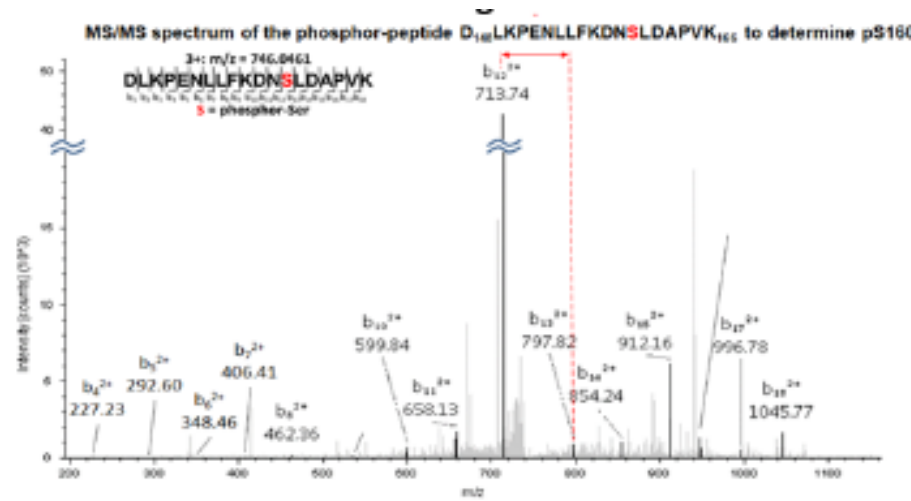

B

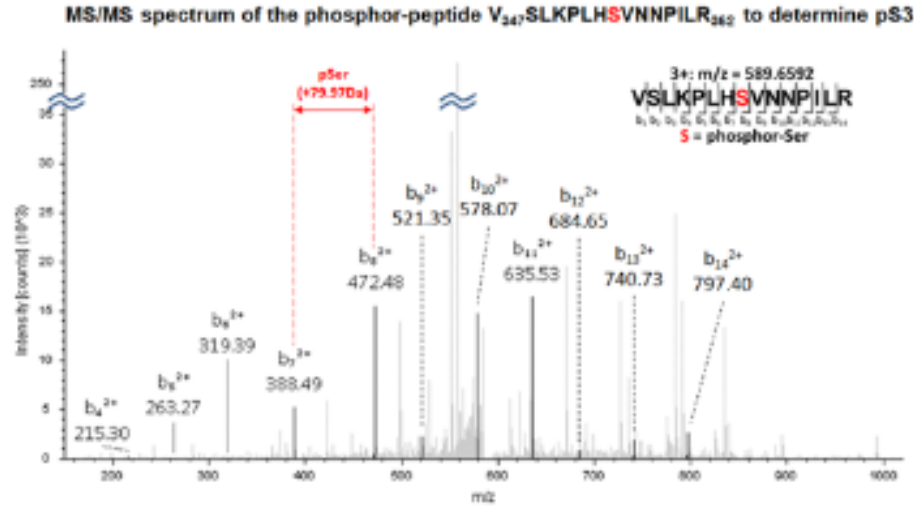

C

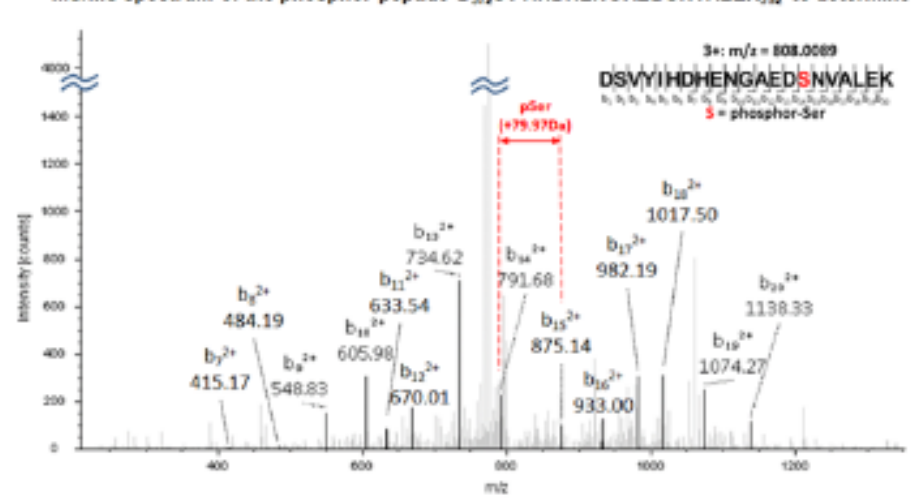

Figure 5

TLK1 Phosphorylates MK5 in vitro on three unique residues. Spectral analysis of MK5 phosphoresidue A) S160, B) S354, and C) S386 determination by MS. 


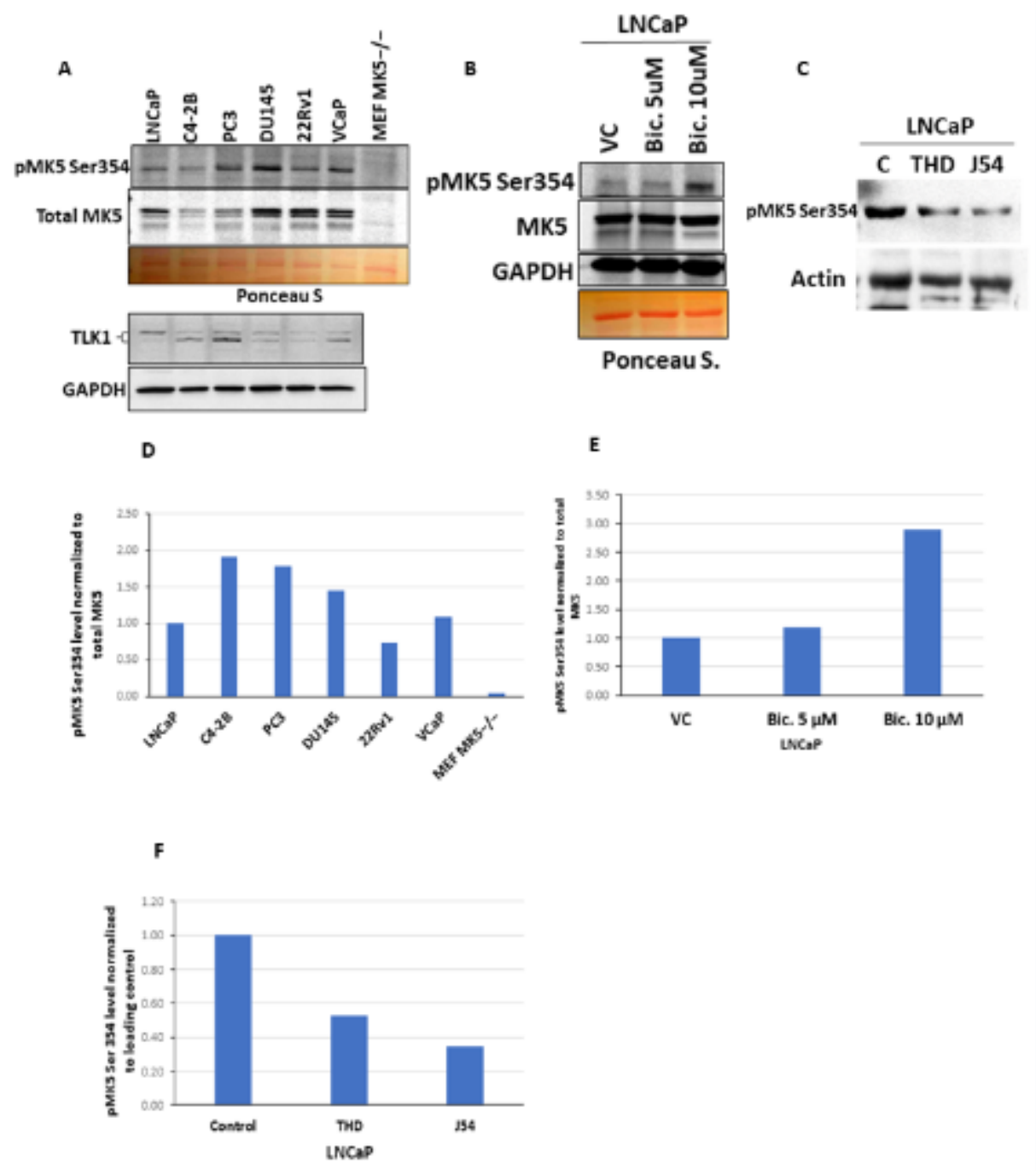

Figure 6

A) pMK5 S354, total MK5, and TLK1 level in different androgen dependent and independent PCa cell lines determined by WB. GAPDH was used as a loading control. B) pMK5 Ser354 level in LNCaP cells after two different concentration of bicalutamide treatment compared to the vehicle control (VC) determined by WB. C) pMK5 S354 level in LNCaP cells after two different TLK1 inhibitor (THD and J54) treatment at 10 $\mu \mathrm{M}$ concentration. D) Densitometric quantification of pMK5 S354 level normalized to total MK5 in different PCA cell lines. E) Densitometric quantification of pMK5 S354 level normalized to total MK5 upon bicalutamide treatment. F) Densitometric quantification of pMK5 S354 level normalized to loading control upon two different TLK1 inhibitor treatment. 

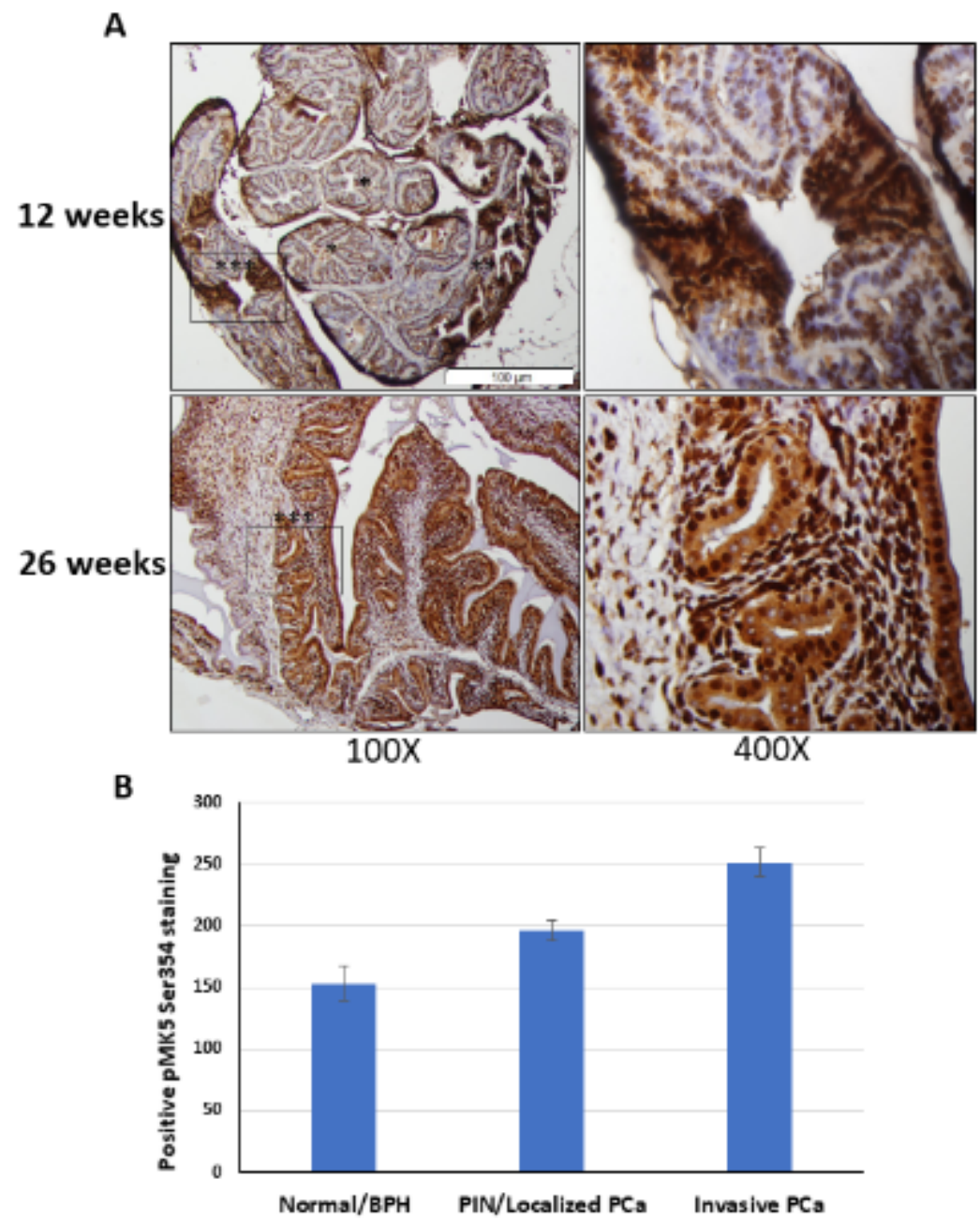

Figure 7

IHC staining revealed elevated pMK5 Ser354 level in PCa progression of TRAMP mice. A) Top panel, pMK5 Ser354 staining in a mixture of normal tissue/BPH, PIN/localized PCa, and invasive PCa regions in 12-weeks old mice. Bottom panel, pMK5 Ser354 staining in the invasive PCa lesions of 26-weeks old mice that has spread throughout the gland. ${ }^{*}=$ normal tissue/BPH, ${ }^{* *}=\mathrm{PIN} /$ localized $\mathrm{PCa}$, and ${ }^{* \star *}=$ invasive PCa. Black dashed boxes represent the magnified regions. Scale bar: $100 \mu \mathrm{m}$. B) ImageJ analysis of the positive staining of pMK5 Ser354 in different regions of TRAMP prostate tumor. 


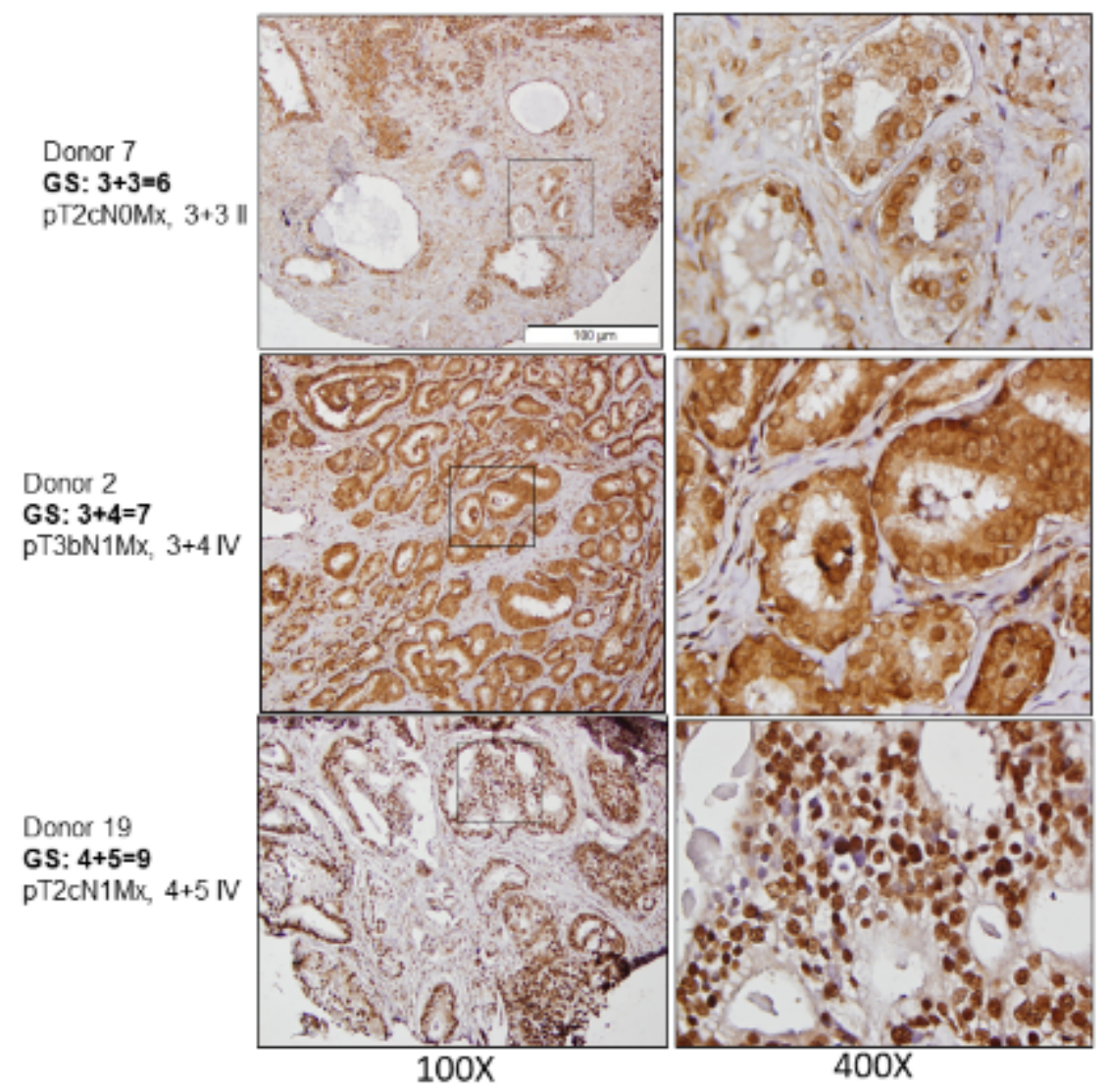

\section{Figure 8}

IHC staining revealed elevated pMK5 Ser354 level in patients with high grade metastatic prostate cancer. pMK5 Ser354 staining in TMA samples of Gleason scores 6, 7, and 9. Black dashed boxes represent the magnified regions. Scale bar: $100 \mu \mathrm{m}$. 

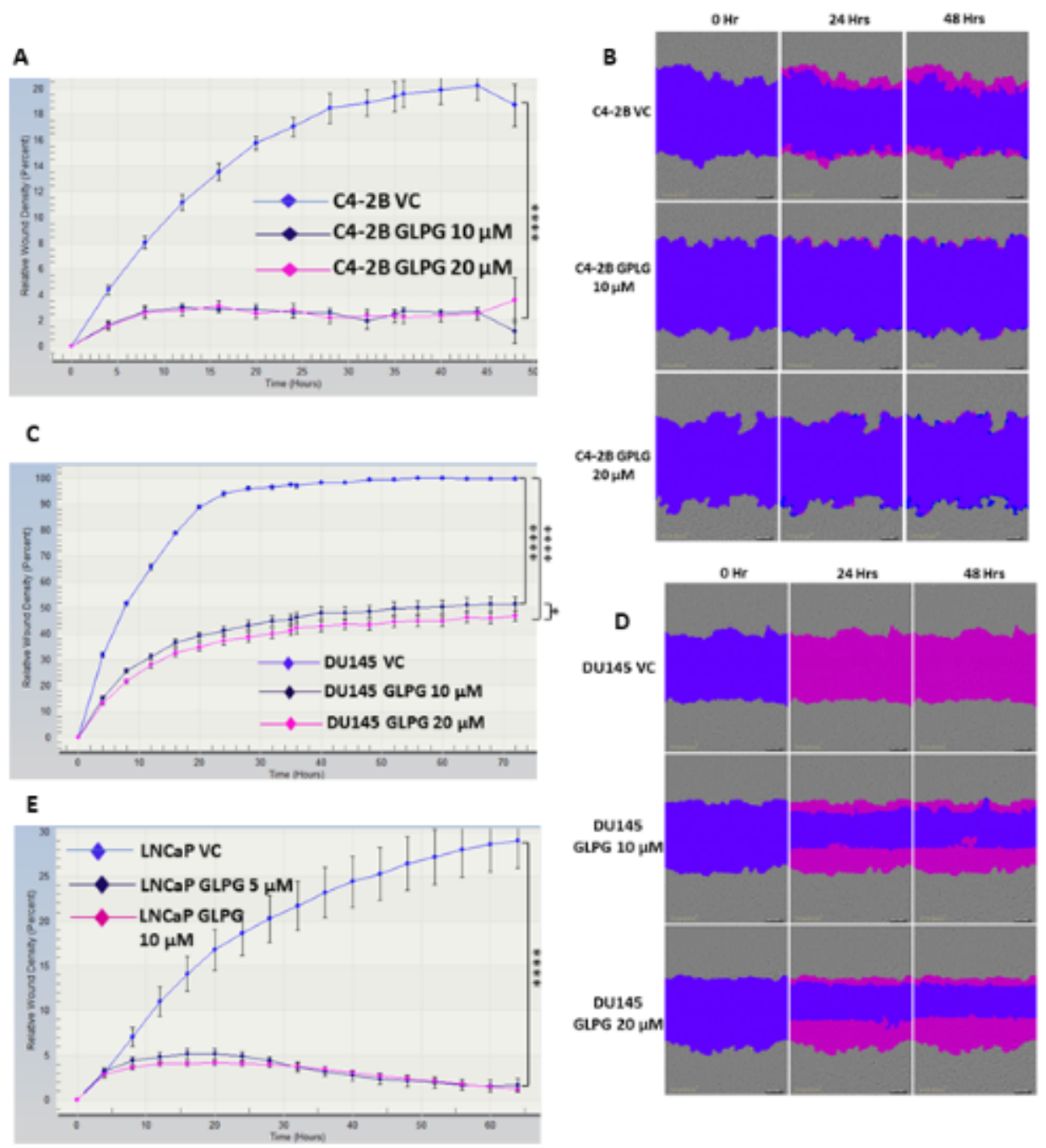

\section{Figure 9}

MK5 inhibition reduces wound healing in PCa cell lines. A, C, E) Scratch wound repair assay was conducted to determine the $2 \mathrm{D}$-migration rate by plotting relative wound density against different time points- A) C4-2B Vehicle control (VC), MR: 1.92 \pm 0.09 ; C4-2B GLPG $10 \mu \mathrm{M}$, MR: 0.31 \pm 0.03 ; and C4-2B GLPG $20 \mu \mathrm{M}$, MR: 0.29 \pm 0.03 cells. C) DU145 VC, MR: 10.45 \pm 0.06; DU145 GLPG $10 \mu \mathrm{M}$, MR: 4.99 \pm 0.18 ; and DU145 GLPG $20 \mu \mathrm{M}$, MR: $4.3 \pm 0.20$ cells. E) LNCaP VC, MR: 2.19 \pm 0.20 ; LNCaP GLPG $5 \mu \mathrm{M}, \mathrm{MR}$ : $0.33 \pm 0.05$; and LNCaP GLPG $10 \mu \mathrm{M}, \mathrm{MR}: 0.32 \pm 0.06$ cells. B, D) Image representation of the wound healing in scratch wound repair assay of different concentration of MK5 inhibitor (GLPG0259) treated B) C4-2B and D) DU145 cells at different time points. ${ }^{*}=p<0.05$ and $* \star \star \star=p<0.0001$. 
A
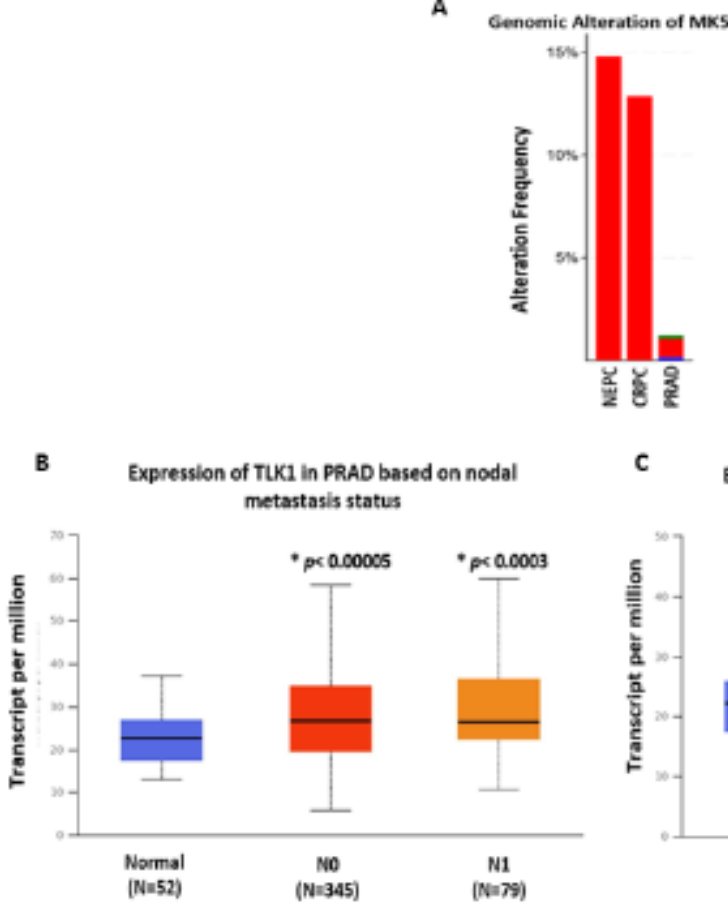

C Expression of MKS in PRAD based on nodal
metastasis status

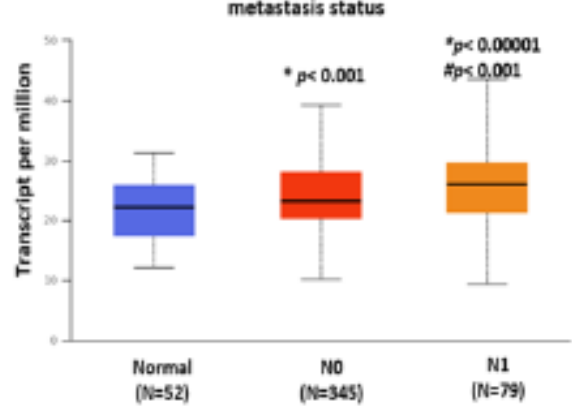

Expression of TLK1 in PRAD based on patient's gleason score

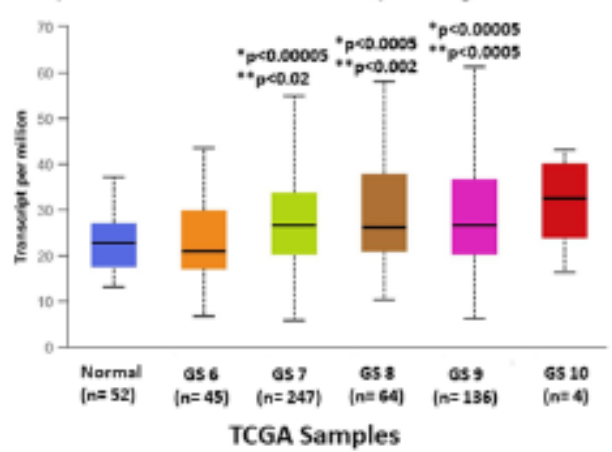

E

Expression of MAPKAPK5 in PRAD based on patient's

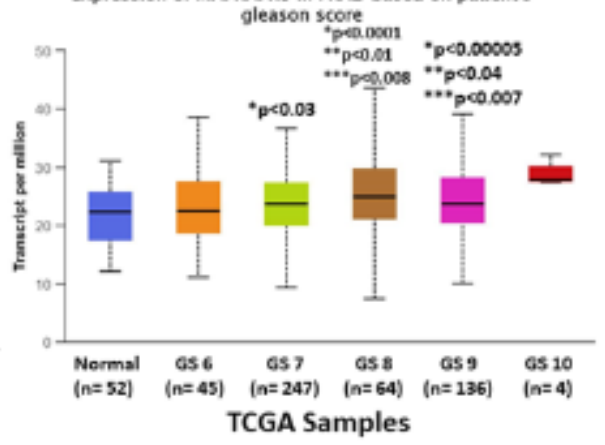

Figure 10

Copy number increase and upregulation of TLK1 and MK5 in metastatic PCa. Bioinformatic analysis of PCa datasets to determine: (A) genomic alteration of MK5; (B) mRNA expression of TLK1 based on nodal metastasis; (C) mRNA expression of MK5 based on nodal metastasis; (D) mRNA expression of TLK1 based on tumor Gleason score; (E) mRNA expression of MK5 based on tumor Gleason score. CRPC= Castration-resistant prostate cancer, $\mathrm{PRAD}=$ Prostate adenocarcinoma, NEPC= neuroendocrine prostate cancer. $\mathrm{N} 0=$ No regional lymph node metastasis, $\mathrm{N} 1=$ Metastases in 1 to 3 axillary lymph nodes. GS= Gleason score. * = comparison with normal, \#= comparison with N0, $* *=$ comparison with GS $6, * \star \star=$ comparison with GS 7. 


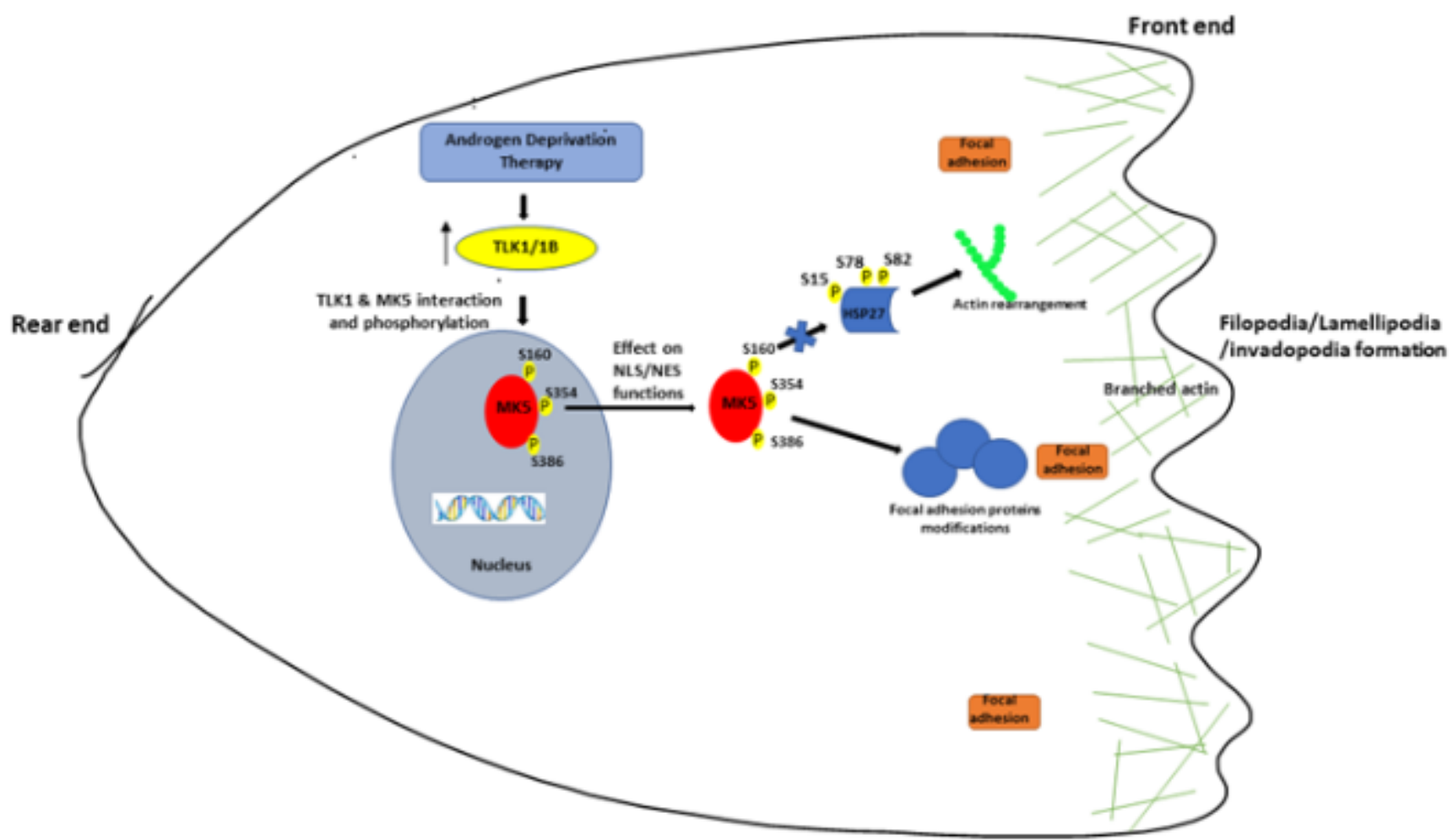

Figure 11

Putative mechanisms of motility promotion in PCa cells by TLK1-MK5 signaling.

\section{Supplementary Files}

This is a list of supplementary files associated with this preprint. Click to download.

- SupplementaryTable23.xlsx

- TLK1MK5SupplemantrayFigures.pdf 